\title{
A Lifelong Model for the Female Reproductive Cycle with an Antimüllerian Hormone Treatment to Delay Menopause ${ }^{\dagger}$
}

\author{
Alison Margolskee ${ }^{1}$ and James F. Selgrade ${ }^{2}$ \\ ${ }^{1}$ Department of Mathematics, North Carolina State University, Raleigh, \\ NC 27695, USA, amargol@ncsu.edu \\ ${ }^{2}$ Department of Mathematics and Biomathematics Program, North Carolina State \\ University, Raleigh, NC 27695, USA, selgrade@math.ncsu.edu
}

\begin{abstract}
A system of 16 nonlinear, delay differential equations with 66 parameters is developed to model hormonal regulation of the menstrual cycle of a woman from age 20 to age 51 . This mechanistic model predicts changes in follicle numbers and reproductive hormones that naturally occur over that time span. In particular, the model illustrates the decline in the pool of primordial follicles from age 20 to menopause as reported in the biological literature. Also, model simulations exhibit a decrease in antimüllerian hormone (AMH) and inhibin $\mathrm{B}$ and an increase in FSH with age corresponding to experimental data. Model simulations using the administration of exogenous AMH show that the transfer of non-growing primordial follicles to the active state can be slowed enough to provide more follicles for development later in life and to cause a delay in the onset of menopause as measured by the number of primordial follicles remaining in the ovaries. Other effects of AMH agonists and antagonists are investigated in the setting of this model.
\end{abstract}

Key Words. Menstrual cycle, primordial follicle, parameter identification

AMS 2000 Mathematics Subject Classification: 92C30, 37N25

† Research supported by NSF grants DMS-0920927 and DMS-1225607.

2 Corresponding author, e-mail: selgrade@math.ncsu.edu, phone: 1-919-515-8589, fax: 1-919-513-7336, postal address: Box 8205, NCSU, Raleigh, NC 27695, USA 


\section{Introduction.}

Regulation of the reproductive cycle in adult women involves hormones produced by the hypothalamic-pituitary-ovarian axis (see Figure 1). The pituitary, prompted by signals from the hypothalamus, secretes follicle stimulating hormone (FSH) and luteinizing hormone (LH) which control ovarian follicle development and ovulation (Yen [1]). The ovaries produce estradiol (E2), progesterone (P4), inhibin A (InhA) and inhibin B (InhB) which affect the synthesis and release of FSH and LH (Karsch et al. [2]). The ovaries also produce antimüllerian hormone $(\mathrm{AMH})$ which affects early follicular development (Skinner [3], Durlinger et al. [4]). As a woman ages, her ability to produce offspring decreases because of decreasing follicle numbers and changes in reproductive hormones (Broekmans et al. [5]). Peak fertility occurs between the ages of 20 and 30 (Soules et al. [6]). By the average age of 41, a woman is considered infertile because conception often takes longer than 12 months [5]. However, in North America and Europe more women are postponing childbearing until their 30's and must deal with the consequences of reduced natural fertility. A mathematical model for hormonal regulation of the menstrual cycle throughout a woman's reproductive life would be useful for studying agerelated changes in menstrual cyclicity. Such models may help to identify parameter variations which are associated with subtle hormonal variations occurring in women in their 30's and model simulations may assist in the testing of hormonal therapies.

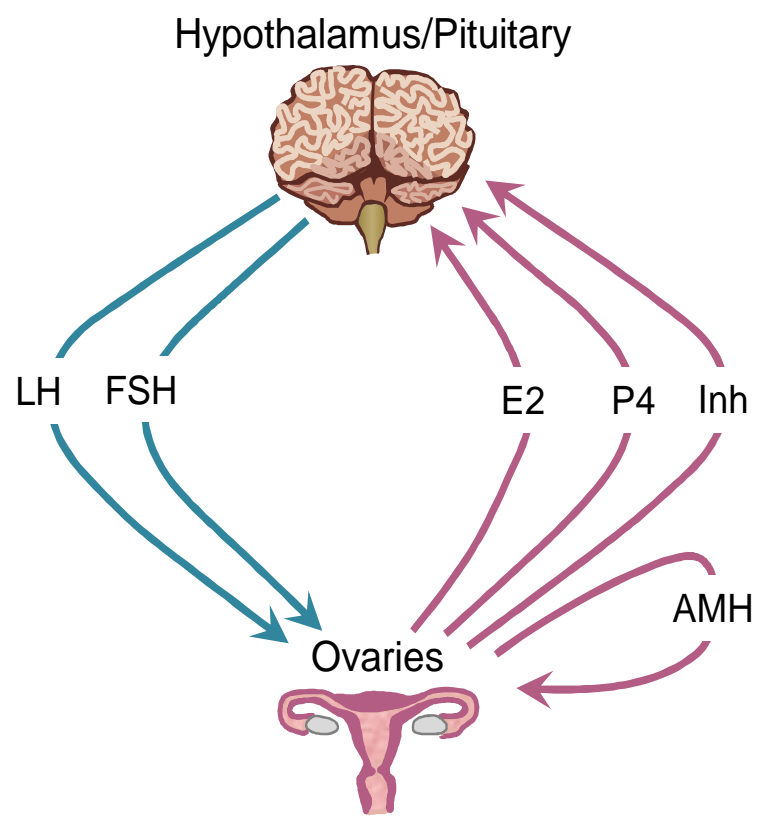

Figure 1: Hormonal control of the menstrual cycle via the hypothalamic-pituitary-ovarian axis. Luteinizing hormone ( $\mathrm{LH})$ and follicle stimulating hormone (FSH) are produced by the pituitary. Estradiol (E2), progesterone $(\mathrm{P} 4)$, the inhibins and antimüllerian hormone $(\mathrm{AMH})$ are produced by the ovaries. 
Differential equations have been used to model different aspects of hormonal control of the menstrual cycle, e.g., see Bogumil et al., 1972a, 1972b [7, 8], Plouffe and Luxenberg, 1992 [9], Selgrade and Schlosser, 1999 [10], Schlosser and Selgrade, 2000 [11], Harris-Clark et al., 2003 [12], Reinecke and Deuflhard, 2007 [13], Pasteur, 2008 [14] and Margolskee and Selgrade, 2011 [15]. These studies modeled the phenomenon on the time scale of days and months, and could model women of various ages by using different parameter sets. Here we develop a variation on these models with the goal to simulate key hormonal changes with advancing age, using a single parameter set to represent women of different ages.

We present a system of 16 delay differential equations with 66 parameters which models a woman's reproductive years between age 20 and 51 from the point of view of hormonal control. Our model simulations approximate data in the literature (Welt et al. [16]) for two age groups of women, 20-34 years old and 35-46 years old. Our model reflects changes in hormone levels and follicle numbers that occur during that time span, e.g., the continual drop in AMH (see [17, 18]), the decrease in InhB between ages 35 and 45 (see [16]), and the subsequent rise in follicular phase FSH, while E2 and P4 levels remain unaffected [19]. The biological mechanism which initiates these changes is the gradual loss of primordial follicles (Skinner [3]). The "primordial pool" refers to the dormant follicles that a woman is born with and this pool continually decreases over time due to atresia or due to transfer to the active state. White et al. [20] recently reported the discovery of stem cells in a woman's ovaries which may produce oocytes after birth. However, we model primordial follicles formed only before birth, their transfer to growing follicles and then these follicles as they mature through primary, preantral, antral and dominant status followed by ovulation and luteinization. Our previous models [10, 12, 15] did not include state variables representing primordial, primary, preantral and small antral follicles nor did they include AMH and InhB. The decline of the primordial pool is eventually realized in a decreased number of preantral and small antral follicles, which directly translates to decreased levels of $\mathrm{AMH}$ and InhB (which are produced by these follicles), and the decreased InhB causes increased FSH production (since InhB inhibits FSH production). A goal of our modeling endeavor is to investigate possible hormonal treatments which may improve the fertility of women in their 30's and early 40's. To this end, we show that the administration of exogenous AMH mitigates the loss of primordial follicles and, hence, provides more and possibly healthier follicles for development later in life.

Section 2 develops the model under study and describes the model system of 16 differential equations (S1)-(S16). We devise an ad hoc procedure for estimating the 66 model parameters and discuss aspects of this procedure in Section 3 and in Appendix A. The resulting parameter sets are included in Appendix B. Results of model simulations are presented in Section 4 with comparisons to data in the biological literature. Statistical comparisons are made between model simulations of hormones and ovarian stages for ages 30 and 40. Section 5 demonstrates how exogenous AMH inputs, AMH agonists and AMH antagonists affect model behavior. Fi- 
nally, we summarize and discuss the results.

\section{Biological Background and Model Development.}

The menstrual cycle of a normally cycling adult female ranges from 25 to 35 days in duration (Ojeda [21]) and consists of the follicular phase, ovulation and then the luteal phase. Pulses of FSH and LH are secreted by the pituitary in response to pulses of gonadotropin-releasing hormone $(\mathrm{GnRH})$ produced by the hypothalamus on a time scale of minutes. Because the ovaries respond to average daily blood levels (Odell [22]), our model tracks average daily concentrations of FSH and LH, lumping the effects of the hypothalamus and the pituitary together and just considering the synthesis and release of FSH and LH on the time scale of days. Models with this simplification have predicted quite well daily hormone data in the literature, e.g., Harris-Clark et al. [12], Pasteur [14] and Margolskee and Selgrade [15]. As part of their normal function, the ovaries produce E2, P4, InhA and InhB which control the pituitary's synthesis and release of the gonadotropin hormones during the various stages of the cycle (Yen [1]). The ovaries also produce AMH which affects early follicular development (Skinner [3]). Here we extend previous models for monthly cycling to the reproductive life span of a woman by beginning at the primordial stage of follicle development and continuing through primary, preantral, and small antral stages (see Figure 2).

The follicular stages in our model in developmental order are primordial (Primor), primary (Primar), preantral follicles $(\operatorname{Pr} A n F)$, small antral follicles $(S m A n F)$, recruited follicles $(R e F)$, growing follicles $(G r F)$, the dominant follicle $(D o m F)$, ovulatory follicle $(O v)$, and four luteal stages $\left(\right.$ Lut $_{1}$ through $\left.L u t_{4}\right)$. Note that our previous models referred to the dominant follicle as primary, but this use of the term primary was not in agreement with biological references, e.g., Skinner [3] and Hansen et al. [23]. Figure 2 depicts the stages of follicular development, the hormones produced by each stage, and which stages are affected by the pituitary hormones LH and FSH.

A primordial follicle consists of an oocyte surrounded by squamous (flat) granulosa cells. If the primordial follicle does not atrophy, it passes to the primary stage where granulosa cells become cuboidal and theca cells are recruited. The primary stage is considered the initial stage of follicular growth $([3,24,25])$, although Hansen et al. [23] referred to primary follicles as non-growing because their growth is gonadotropin independent. The transition from the primordial to the primary is stimulated and inhibited by a variety of ovarian factors (Skinner [3] and Reddy et al. [26]). Skinner [3] discussed granulosa and theca cell products that promote the primordial to the primary transition such as KL, KGF and bFGF growth factors. On the other hand, the hormone AMH produced by primary, preantral and small antral follicles is known to inhibit the transition from the primordial to the primary pool (Skinner [3] or Reddy et al. [26]). Also Reddy et al. [26] described ovarian genetic factors such as oocyte PTEN and Foxo3a which suppress the activation of the primordial follicle pool and hence the transition to the primary pool. The first ovarian stage in our model, Primor, represents the primordial 


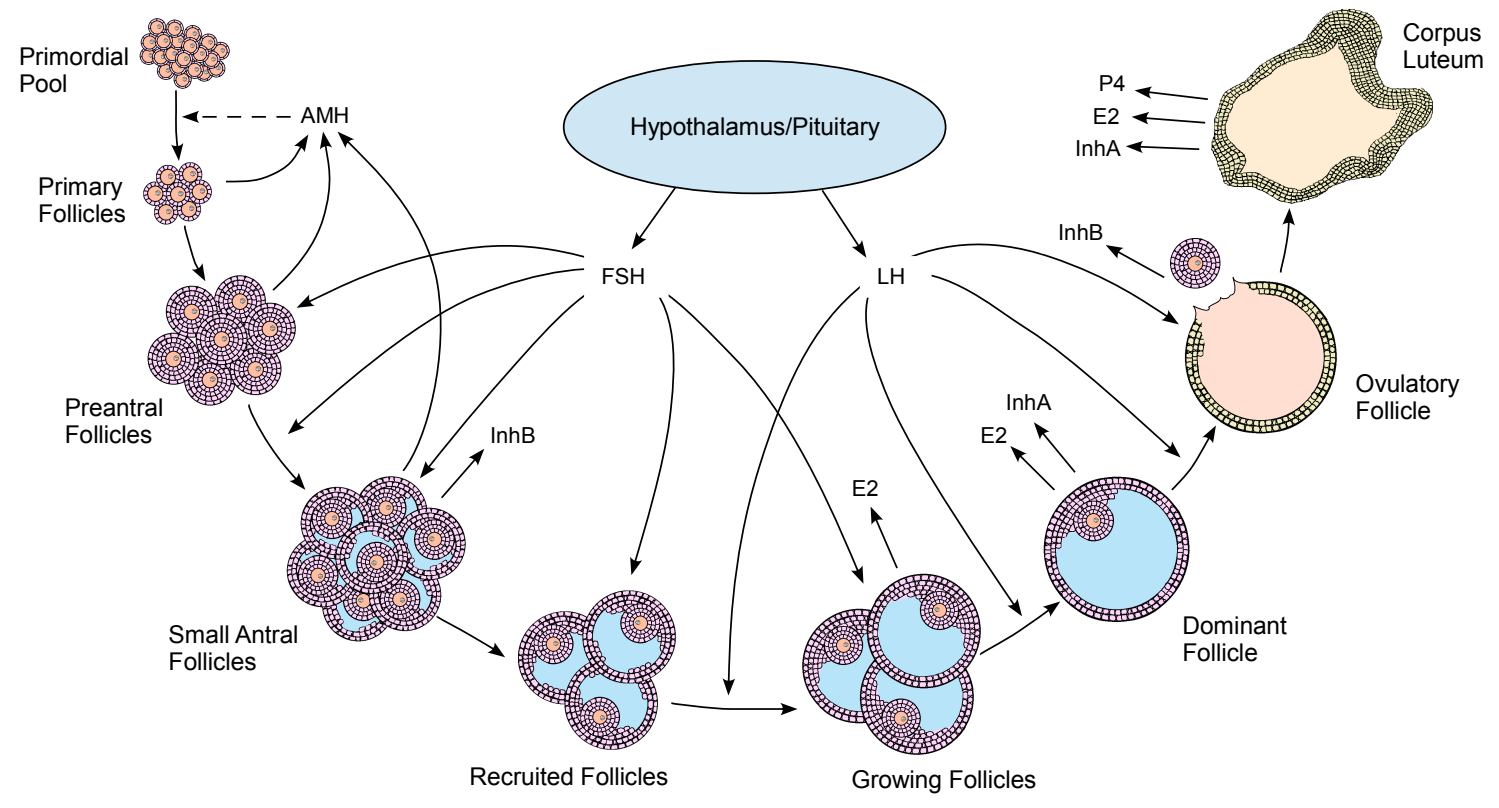

Figure 2: Depicted are the stages of follicular development as modeled by our system of equations. The follicular stages included in our model are primordial follicles, primary follicles through the ovulatory follicle and corpus luteum. Arrows between each stage represent a transition from one follicle type to the next. Arrows pointing away from follicles represent hormones secreted by these follicles. FSH and LH are produced and released by the Hypothalamus/Pituitary. Arrows pointing from FSH and LH represent the effects of these hormones on follicle growth and transition. The dashed arrow pointing from AMH indicates the inhibitory role that AMH plays on the primordial to primary transition. 
pool of follicles. The differential equation for this stage is a single term representing the decay rate of the primordial pool and is directly proportional to Primor and inversely proportional to both Primor and $A M H$ (see equation (S1)). This term models inhibitory signals between primordial follicles and the inhibitory role of AMH on the primordial to primary transition. The decay term from (S1) appears as a growth term in (S2) for the number of primary follicles, Primar. The factor of $r_{\text {surv }}$ represents the fraction of primordial follicles that are not lost to atresia before becoming primary follicles, i.e., $r_{\text {surv }}$ is the survival rate. The amount of $A M H$ in (S1)-(S2) is given by (A5) appearing below.

$$
\begin{aligned}
& \frac{d}{d t} \text { Primor }=-\frac{r_{1} \text { Primor }}{1+c_{\text {prm }} \text { Primor }+c_{A M H} A M H} \\
& \frac{d}{d t} \text { Primar }=r_{\text {surv }} \frac{r_{1} \text { Primor }}{1+c_{\text {prm }} \text { Primor }+c_{A M H} A M H}-r_{2} \text { Primar }
\end{aligned}
$$

The Primar stage is followed by $\operatorname{Pr} A n F$ and $S m A n F$ (equations (S3)-(S4)), which represent preantral and small antral follicles, respectively. These stages, and all subsequent follicular stages, represent volumes instead of numbers of follicles. Thus, we multiply the transfer term from Primar to $\operatorname{Pr} A n F$ in (S3) by a parameter for the average volume per preantral follicle (vol2). A follicle which ultimately releases its ovum spends several months [27] developing from a preantral follicle into an ovulatory follicle, $O v$ in (S8). During that time the maturing follicle acquires FSH receptors and its future growth becomes gonadotropin dependent as indicated by the decay term in (S3), and the growth terms in (S4). These terms have the form of an increasing Hill function of FSH in agreement with Zeleznik [28] who suggested that FSH levels must rise above a threshold to initiate follicular development. Thus the sustained growth of the small antral stage, $S m A n F$ in (S4), depends on FSH attaining a threshold serum concentration. The exponents $\alpha$ and $\beta$ are referred to as Hill coefficients and we determine these parameter values through our estimation procedure.

$$
\begin{aligned}
& \frac{d}{d t} \operatorname{PrAnF}=\operatorname{vol} \cdot r_{2} \cdot \operatorname{Primar}-r_{3} \frac{F S H^{\alpha}}{K m_{F 1}^{\alpha}+F S H^{\alpha}} \operatorname{Pr} A n F \\
& \frac{d}{d t} S m A n F=r_{3} \frac{F S H^{\alpha}}{K m_{F 1}^{\alpha}+F S H^{\alpha}} \operatorname{Pr} A n F+\left[r_{4} \frac{F S H^{\beta}}{K m_{F 2}^{\beta}+F S H^{\beta}}-r_{5}\right] S m A n F
\end{aligned}
$$

At the beginning of a woman's monthly cycle, 6 to 12 follicles are recruited from the pool of early antral follicles to grow under the influence of FSH and LH with the opportunity to 
reach ovulatory size (Figure 2). The growth of the recruited follicles, $R e F$ in (S5), depends on $S m A n F$ and on FSH reaching an early follicular phase threshold, see (S5). AMH is thought to decrease the FSH-sensitivity of late antral follicles (see (S6)), playing a role in the selection of the dominant follicle [25]. Typically one follicle is selected to be dominant and then to release its ovum in response to a surge of LH. Ovulation and luteinization transform the dominant follicle into the corpus luteum which produces $\mathrm{P} 4$ to prepare the endometrium for pregnancy. If fertilization does not occur, the corpus luteum regresses, menstruation follows and a rise in FSH marks the beginning of the next cycle. The state variables in (S5)-(S12) represent tissue volumes of 8 distinct stages of the ovary during the follicular and luteal phases of the cycle (see Harris-Clark et al., [12]). ReF, GrF and DomF denote the recruited follicles, the growing follicles and the preovulatory or dominant follicle, respectively. $O v$ represents a periovulatory stage and $L u t_{i}, i=1, \ldots, 4$, denote four luteal stages. Since clearance from the blood of the ovarian hormones is on a fast time scale, we assume that blood levels of E2, P4, InhA, InhB, and AMH are at quasi-steady state (Keener and Sneyd [29]) as did Bogumil et al. [7]. Hence, we take these concentrations to be proportional to the tissue volumes during the appropriate stages of the cycle giving the 5 auxiliary equations (A1)-(A5).

$$
\begin{aligned}
\frac{d}{d t} \operatorname{ReF} & =r_{5} \operatorname{SmAnF}+\left[c_{1} \frac{F S H^{\gamma}}{K m_{F 3}^{\gamma}+F S H^{\gamma}}-c_{2} L H^{\delta}\right] R e F \\
\frac{d}{d t} G r F & =c_{2} L H^{\delta} R e F+\left[c_{3} \frac{F S H}{1+\frac{A M H}{K i_{A M H}}}-c_{4} L H\right] G r F \\
\frac{d}{d t} D_{o m F} & =c_{4} L H \cdot G r F-c_{5} L H^{\omega} D o m F \\
\frac{d}{d t} O v & =c_{5} L H^{\omega} D_{o m F}+c_{6} L H \cdot D o m F-c_{7} O v \\
\frac{d}{d t} L_{u} t_{1} & =c_{7} O v-k_{1} L u t_{1} \\
\frac{d}{d t} \text { Lut }_{2} & =k_{1} \text { Lut }_{1}-k_{2} \text { Lut }_{2} \\
\frac{d}{d t} \text { Lut }_{3} & =k_{2} \text { Lut }_{2}-k_{3} \text { Lut }_{3} \\
\frac{d}{d t} \text { Lut }_{4} & =k_{3} \text { Lut }_{3}-k_{4} \text { Lut }_{4} .
\end{aligned}
$$


In terms of these stages, the ovarian hormones are given by:

$$
\begin{aligned}
& E_{2}=e_{0}+e_{1} G r F+e_{2} D o m F+e_{3} L_{u t_{4}} \\
& P_{4}=p_{0}+p_{1} \text { Lut }_{3}+p_{2} \text { Lut }_{4} \\
& \operatorname{Inh} A=h_{0}+h_{1} \text { DomF }+h_{2} L_{u t_{2}}+h_{3} L_{u t_{3}} \\
& \operatorname{InhB}=j_{0}+j_{1} S m A n F+j_{2} O v \\
& A M H=a_{1} \text { Primar }+a_{2} \operatorname{Pr} A n F+a_{3} S m A n F
\end{aligned}
$$

The ovarian hormones regulate the synthesis and release of FSH and LH by the hypothalamus and pituitary as described by four differential equations (S13)-(S16), which are similar to the equations in Harris-Clark et al. [12]. The state variables $R P_{L H}$ and $R P_{F S H}$ represent the amounts of these hormones in the pituitary and $L H$ and FSH represent the blood concentrations of these hormones. The biological literature (Karsch et al. [2], Liu and Yen [30] or Yen [1]) indicates that LH exhibits a biphasic response to E2, with low concentrations of E2 inhibiting and high levels of E2 stimulating LH serum concentrations. To capture this our model assumes that the effect of E2 on LH synthesis is different than the effect on LH release [11], i.e., E2 inhibits release (see the denominator of the second term in (S13)) but at high levels E2 promotes synthesis (see the Hill function in the numerator of the first term of (S13)). On the other hand, P4 inhibits LH synthesis but promotes release [11]. The release term appears in (S13) as a decay term and in (S14) as a growth term, where it is divided by blood volume $v$. The equations (S15)-(S16) for FSH are similar except the synthesis term has InhA and InhB inhibition. Because hormone synthesis is biochemically more complicated than release, the time-delay parameters $d_{E}, d_{P}, d_{\operatorname{Inh} A}$ and $d_{\operatorname{Inh} B}$ are assumed only for the synthesis terms and describe the periods between the time when changes in serum levels of E2, P4 and Inh occur and the time when subsequent changes in LH and FSH synthesis rates occur. Based on results of previous work $[12,15,11]$, a Hill coefficient of 8 provides the appropriate steepness for the LH synthesis curve in (S13) so that simulations will closely approximate LH data in the literature $[16,31]$. 


$$
\begin{aligned}
\frac{d}{d t} R P_{L H} & =\frac{V_{0, L H}+\frac{V_{1, L H} E_{2}^{8}\left(t-d_{E}\right)}{K m_{L H}^{8}+E_{2}^{8}\left(t-d_{E}\right)}}{1+P_{4}\left(t-d_{P}\right) / K i_{L H, P}}-\frac{k_{L H}\left[1+c_{L H, P} P_{4}\right] R P_{L H}}{1+c_{L H, E} E_{2}} \\
\frac{d}{d t} L H & =\frac{1}{v} \frac{k_{L H}\left[1+c_{L H, P} P_{4}\right] R P_{L H}}{1+c_{L H, E} E_{2}}-c l_{L H} L H \\
\frac{d}{d t} R P_{F S H} & =\frac{V_{F S H}}{1+\frac{I n h A\left(t-d_{I n h A}\right)}{K i_{F S H} \text { InhA }}+\frac{I n h B\left(t-d_{I n h B}\right)}{K i_{F S H, I n h B}}}-\frac{k_{F S H}\left[1+c_{F S H, P} P_{4}\right] R P_{F S H}}{1+c_{F S H, E} E_{2}^{2}} \\
\frac{d}{d t} F S H & =\frac{1}{v} \frac{k_{F S H}\left[1+c_{F S H, P} P_{4}\right] R P_{F S H}}{1+c_{F S H, E} E_{2}^{2}}-c l_{F S H} F S H
\end{aligned}
$$

\section{Methods.}

\subsection{Computational methods}

Numerical computations are performed using Matlab version 7.12 on a quad-core PC equipped with a 7th generation Intel chip and 8.00 GB installed RAM. Delay differential equations are solved using Matlab's built-in delay differential equations solver dde23, which numerically integrates delay differential equations with constant delays. It employs the Runge Kutta $(2,3)$ pair to perform a variable step integration, and uses a Hermite cubic interpolant to determine lagged values from stored history [32]. When equations are decoupled, and the result produces a smaller system of equations with no delays, this system is integrated using Matlab's ode23.

Numerical drift can result when the local truncation errors of a numerical approximation are compounded with each iteration, causing the numerical approximation to drift away from the true solution [33]. Numerical drift resulting during numerical integration will accumulate over longer periods of integration. The extent of numerical drift is dependent upon the step size used during integration, with smaller step sizes resulting in less numerical drift. Thus, the extent of numerical drift can be analyzed with a grid-refinement study. For periodic solutions of differential equations, numerical drift can present itself as a phase shift in the time direction. Since solutions of our system that are of interest are exponentially attracting, we expect to see numerical drift primarily in the time direction.

To determine the extent of numerical drift of Runge Kutta methods on our model, we performed a grid-refinement study using a fixed step 4th order Runge Kutta method for delay differential equations which we coded and implemented in Matlab. We refer to this integrator 


\begin{tabular}{l|l}
$\begin{array}{l}\text { Step size } \\
\text { (Days) }\end{array}$ & $\begin{array}{l}\text { Phase Shift } \\
\text { (Days) }\end{array}$ \\
\hline 0.1 & 0 \\
0.05 & 0.2161 \\
0.025 & 0.2415 \\
0.0125 & 0.2436 \\
\hline
\end{tabular}

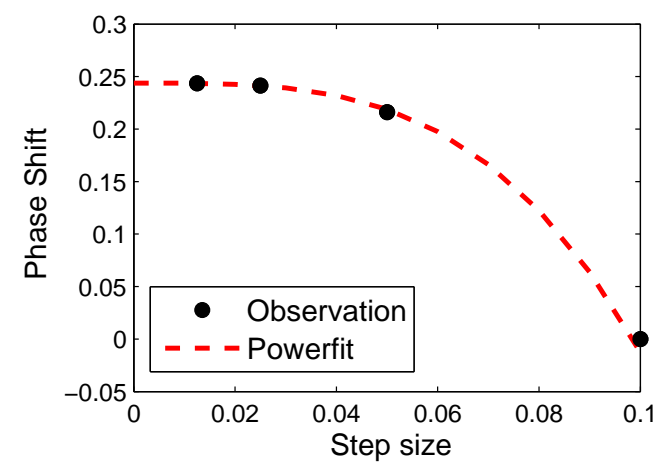

Table 1: Summary of grid-refinement study of phase shift in time direction of numerical simulation after integrating from age 20 to age 50 using ddeRK4. Column 1 is the step size used in integration, Column 2 is the difference in day of LH peak (phase shift) at the end of 30 years when compared to the solution obtained using the step size of 0.1 days. Fitting a simple power function $\left(a+b x^{c}\right)$ to these data gives an intercept of $a=0.2438$ days, which implies a phase shift for the simulation with step size 0.1 approaches 0.2438 days with successively smaller step sizes.

as ddeRK4 [34]. The solutions obtained by using different time steps in the integrator ddeRK4 were compared to determine the extent of numerical drift. We found that a step size of 0.1 days limits the numerical drift in the time direction to less than 0.25 days when the model is integrated over a period of 30 years, from age 20 to age 50 (see Table 1). When the phase shift in the time direction is accounted for, i.e. the solution profiles are centered at the $L H$ peak, then a time step of 0.1 days limits the deviation in the solution profiles to less than $0.5 \%$ for integration spans of more than 30 years. In an analogous grid refinement study, the numerical drift in the noncycling stages (Primor and Primar) was observed. These stages are affected by numerical drift to a lesser extent than the monthly cycling stages. The numerical drift in the solution profiles for Primor and Primar can be limited to less than $0.1 \%$ across the 30 year span by using a stepsize of 80 days during integration.

The longest time span of integration reported here is from age 20 to age 51, but only the noncycling equations (Primor and Primar, see equations (S1) and (S2)) are integrated for this time span (see Section 3.8). These equations also contain no delay, so they are simulated using Matlab's ode integrator ode23. The numerical drift in the magnitudes of these noncycling equations can be limited to less than $0.1 \%$ when a step size of 80 days is used. So we integrate this small system using ode23 with the option MaxStep set to 80 .

The monthly cycling differential equations (Equations (S3) through (S16)) are simulated for two-month time spans (see Section 3.8). The numerical drift in the time direction over this short time span is negligible. The numerical drift in the magnitude of the solution profiles can be limited to less than $0.5 \%$ by using a time step of 0.1 days. So we integrate these equations using dde23 with the option MaxStep set to 0.1. 


\subsection{Parameter Identification (PID)}

Estimating the 66 parameter values in system (S1)-(S16) and auxiliary equations (A1)-(A5) requires multiple data sets and considerations of parameter sensitivity and correlation. Data are available in the biological literature for blood levels of the pituitary and ovarian hormones but not for the state variables of eqs. (S3)-(S12). However, some information is known about realistic values for volumes of ovarian stages (e.g., Nussey and Whitehead [27]). Attempting to be faithful to the biology and to use valid numerical techniques leads us to develop an ad hoc, iterative procedure for estimating the parameters. Some details of our process are described in the following subsections and in Appendix A and the resulting parameter sets are included in Appendix B.

\subsection{Least squares data fitting}

In least squares data fitting, the objective function to be optimized has the form [35]:

$$
f(\vec{q})=\sum_{j=1}^{M}\left|d^{j}-y\left(t_{j} ; \vec{q}\right)\right|^{2}=R(\vec{q})^{T} R(\vec{q})
$$

where $\vec{q}$ is the parameter vector, $y$ is a single model output and $d$ is the data sampled at $M$ time points. $R$ is a column vector of length $M$ with components $\left(d^{j}-y\left(t_{j} ; \vec{q}\right)\right)$ and is referred to as the residual between the data and the model output. If the output is a vector of observations $\vec{y}$ of length $N$ with components $y_{i}$ then we have

$$
f(\vec{q})=\sum_{j=1}^{M}\left\|\vec{d}^{\vec{j}}-\vec{y}\left(t_{j} ; \vec{q}\right)\right\|_{2}^{2}=\sum_{j=1}^{M} \sum_{i=1}^{N}\left|d_{i}^{j}-y_{i}\left(t_{j} ; \vec{q}\right)\right|^{2}=R(\vec{q})^{T} R(\vec{q})
$$

where $R$ is of length $M N$ with components $\left(d_{i}^{j}-y_{i}\left(t_{j} ; \vec{q}\right)\right)$.

Fitting the model output to data involves finding a $\vec{q}$ that minimizes the function $f(\vec{q})$. There are many optimization algorithms that can be implemented to find a minimum to a least squares objective function. Public domain codes for many of these optimization algorithms can be found online in the Numerical Analysis and Modelling software repository at Zuse Institut Berlin (ZIB) [36]. These codes are based on the algorithms presented in a book by P. Deuflhard [37]. Of these codes we explored the use of NLSQERR, which is an implementation of unconstrained Gauss-Newton with an error oriented convergence criterion. In order to insure that the parameters of our system are positive, we optimize the natural log of the parameter values and then exponentiate after optimization.

\subsection{Data used during PID and model comparison}

Data used during parameter identification are for primordial and primary follicle counts, and plasma concentrations of AMH, E2, P4, InhA, InhB, LH, and FSH. All data are obtained from the literature $[23,38,17,39,40,41,42,16]$. Data for follicle counts and AMH are for ages 
20 to 51 years. Data for E2, P4, InhA, InhB, LH, and FSH are for women between ages 20 and 34 years. Additional data for InhB and FSH are for women between ages 35 and 46 years. For more information on the residuals used during parameter identification see Appendix A.

Hansen et al. (2008) [23] reported data for gonadotropin independent follicle counts. We take these data to represent the sum of primordial and primary follicle counts. The data were reported in a table along with the age of the subjects. The number of subjects between age 20 and 51 years totaled 103. We refer to this data as Hansen data (see Figure 3).

Plasma concentration data for AMH from women of age 20 years to 51 years are taken from several sources $[38,17,39,40,41,42]$. No single clinical data set provided ample samples for all ages. Some of the data sets spanned only a portion of the ages of interest, and all but one of the data sets had sparse sample sizes for most of the ages. Van Beek et al. [41] had data spanning ages 20 to 38 years, each age having a sample size of less than 10. Data from van Disseldorp et al. [42] covered ages 26 to 47, and only 6 of the 22 ages had sample sizes of at least 10. Hagen et al. [38] had data spanning the entire range of interest, however all but one age had sample sizes of 6 or less. Data from Tehrani et al. [40] spanned ages 20 to 50, but only 6 of these ages had sample sizes of at least 10. Data from Sowers et al. [39] was an exception, having a sample size of 50 people for each of the ages reported, however the data set only covered ages 42 to 47 . Combining the data from these six sources results in a data set covering ages 20 to 51, where all but 4 of the ages have sample sizes of at least 10 and most ages have sample sizes greater than 20. AMH data sets were in $\mathrm{ng} / \mathrm{mL}$ except for Hagen which was converted from $\mathrm{pmol} / \mathrm{L}$ to $\mathrm{ng} / \mathrm{mL}$ using the conversion $1 \mathrm{pmol} / \mathrm{L}=7.14 \mathrm{ng} / \mathrm{mL}$ [43]. The resulting data set is a set of average AMH concentrations by age for ages 20 to 51, obtained from a compiled sample of 849 points. We refer to this compiled data as $\mathrm{AMH}_{\text {data }}$ (see Figure 3).

Daily plasma concentrations of E2, P4, InhA, InhB, LH and FSH for women from ages 20-34 ( $\mathrm{n}=23$ ) are taken from Welt et al. (1999) [16], and we use this data to compare the model solved at age 30 . We refer to this data as $\mathrm{E} 22_{\text {data }}, \mathrm{P} 4_{\text {data }}$, etc. InhB and FSH daily plasma concentrations for women from ages 35-46 $(\mathrm{n}=21)$ from Welt et al. (1999) [16] are used to compare the model solved at age 40. We refer to this data as $\operatorname{InhB}_{\text {data,older }}$ and $\mathrm{FSH}_{\text {data,older}}$. We include InhB and FSH data for older women in order to capture the decrease of InhB and increase of FSH with age $[16,19,44]$ that make these hormones markers of reduced ovarian function. We do not include hormones from the older age group that showed no significant difference between the two age groups, i.e. LH and P4 (p-value $>0.05$, not significant) [16]. InhA showed a significant difference $(\mathrm{p}$-value $<0.04)$ between the two age groups at only one data point (between ovulation and the luteal phase) [16], but we did not consider this to be an important characteristic to capture with our model, thus InhA data for the older women is not included in our comparison. Welt et al. [16] noted a significant increase in follicluar phase E2 in the older group as compared with the younger group ( $\mathrm{p}$-value $<0.02$ ), however other sources have cited decreased E2 concentrations in the menopausal transition [5, 45], or 
no significant difference between women of 22-34 years and those of 41-46 years old (p-value > 0.05, not significant) [46]. Thus we do not include E2 concentrations from the Welt data for the older women in our analysis.

\subsection{Sensitivity, correlation, and uncertainty quantification}

The model presented here has a large number of parameters, and a number of state variables for which there are no direct experimental data. Attempting to identify all of the parameters at once leads to poor convergence of the numerical optimization schemes, and limited parameter identifiability. In the presence of poor convergence, it may be helpful to examine the sensitivity of the model to the parameters and correlations among the parameters. Insensitivity of a model to a parameter means that large changes in the parameter have little effect on the model output. This leads to greater uncertainty of the optimal parameter value and can prevent an optimization algorithm from converging. If a pair of parameters is correlated then changing one parameter is related to changing the other parameter, which may limit parameter identifiability.

The sensitivity of a model output $y$ with respect to a parameter set $\vec{q}$ is the matrix $\frac{\partial y}{\partial \vec{q}}$ (also called the Jacobian of $y$ ) evaluated at the time points $t_{1}, t_{2}, \ldots t_{M}$ associated with the data [47],

$$
S(\vec{q})=\frac{\partial y}{\partial \vec{q}}=\left[\begin{array}{cccc}
\frac{\partial y\left(t_{1} ; \vec{q}\right)}{\partial q_{1}} & \frac{\partial y\left(t_{1} ; \vec{q}\right)}{\partial q_{2}} & \ldots & \frac{\partial y\left(t_{1} ; \vec{q}\right)}{\partial q_{p}} \\
\frac{\partial y\left(t_{2} ; \vec{q}\right)}{\partial q_{1}} & \frac{\partial y\left(t_{2} ; \vec{q}\right)}{\partial q_{2}} & & \\
\vdots & & \ddots & \\
\frac{\partial y\left(t_{M} ; \vec{q}\right)}{\partial q_{1}} & & & \frac{\partial y\left(t_{M} ; \vec{q}\right)}{\partial q_{p}}
\end{array}\right]
$$

It describes the sensitivity of the model to the parameters at the chosen time points. Here $p$ is the number of parameters in $\vec{q}$ and $M$ is the number of data points, which makes $S$ a matrix of size $M \times p$. If the model output is a vector $\vec{y}$ of length $N$ then the sensitivity matrix will be a matrix of size $M N \times p$. Note that sensitivities will vary depending on the parameter values, $\vec{q}$, and the times, $t_{1}, \ldots, t_{M}$, at which they are evaluated. For comparison across parameters and outputs of differing magnitudes, it is often helpful to consider the relative sensitivities which are obtained by multiplying each element $\partial y\left(t_{i} ; \vec{q}\right) / \partial q_{j}$ by $q_{j} / y\left(t_{i} ; \vec{q}\right)$ [48]. The five most sensitive parameters in our model according to the regular sensitivities are $c_{6}, c_{L H, E}, c_{2}, c_{F S H, E}$ and $c_{3}$. In contrast, the most sensitive parameters according to the relative sensitivities are $\delta, K m_{L H}$, $c_{1}, r_{5}$, and $c_{L H P}$. This difference in the sensitivity rankings is due to the magnitudes of the parameters. The parameters $c_{L H, E}, c_{F S H, E}, c_{2}$ and $c_{3}$ are smaller in magnitude than many of the other parameters (see Tables B.2 and B.3 in Appendix B), so it is reasonable that a small absolute change in these parameters could result in a larger change in the model output. The 
relative sensitivities effectively look at how the percent change in the parameters affects the model output.

The covariance of parameters can be used to determine correlations among parameters and to quantify the uncertainty in a parameter set. Normally covariance of a set of random variables would be estimated from a sample distribution of the variables, but here we do not have any information about how $\vec{q}$ varies with the model output. We do however have information about how the model output varies with changes in $\vec{q}$, namely we know the sensitivity matrix $S(\vec{q})=\frac{\partial y}{\partial \vec{q}}$.

Given knowledge of the sensitivity matrix, $S(\vec{q})$, the covariance matrix, cov, of the parameters $\vec{q}_{j}$ can be estimated via the unbiased estimator, $\widehat{\operatorname{cov}}(\vec{q})$, given by $[48,49]$

$$
\widehat{\mathrm{COV}}=\sigma^{2}\left(S^{T} S\right)^{-1}
$$

Here the variance, $\sigma^{2}$, is assumed constant and can be estimated as

$$
\widehat{\sigma}^{2}=R^{T}(\vec{q}) R(\vec{q}) /(n-p)
$$

where $R$ is the residual between the model and data, $n$ is the number of data points (length of $R$ ), and $p$ is the number of parameters (length of $\vec{q}$ ). The square root of the diagonal entries of the covariance matrix are the standard errors associated with the parameters, thus the covariance matrix can be used to estimate the uncertainty in the choice of parameters. The covariance matrix can also be used to determine correlations among the parameters. Correlation of parameters can be given by

$$
C_{i j}=\frac{\operatorname{cov}_{i j}}{\sqrt{\operatorname{cov}_{i i} \operatorname{cov}_{j j}}}
$$

where cov is the covariance matrix, and can be estimated using $\widehat{\operatorname{cov}}$ in place of cov.

The correlation matrix $C$ is symmetric with 1's on the diagonal, and values between -1 and 1 elsewhere. The closer the $C_{i j}$ entry is to \pm 1 , the more correlated are the parameters $q_{i}$ and $q_{j}$. A reasonable rule proposed in Olufsen and Ottesen [48] is to consider all entries greater than 0.9 in magnitude to imply correlation. Note that $S^{T} S$ must have full rank in order to be inverted, and since $\operatorname{rank}\left(\left(S^{T} S\right)_{p \times p}\right)=\operatorname{rank}\left(S_{n \times p}\right) \leq \min (n, p)$, it is necessary that $p$ be less than $n$. There need to be at least as many data points as there are parameters. If there are too many parameters compared to data, then there will necessarily be correlations among the parameters. If a pair of parameters is determined to be correlated, it may be possible to decouple the parameters or reduce the parameter set through nondimensionalization [50].

\subsection{Model-specific correlations}

The parameter $r_{\text {surv }}$ shows correlations with $a_{1}$ and $r_{2}$ (see Eqs. (S1)-(S2) and (A5)), with correlation coefficients of 0.938 and 0.997 , respectively. Thus we estimate $r_{\text {surv }}$, and fix it during optimization. The parameter $r_{\text {surv }}$ is estimated from the decline of Hansen data and the 
estimated monthly pool of primary follicles. The approximate decline of the primordial pool from at age 20 is 22,000 per year (taken as the slope of the power fit to data in Hansen et al. [23]), or approximately 1833 per month. According to Nussey and Whitehead (2001) [27], it takes about 120 days ( 4 months) for a new primary follicle to reach the preantral stage $(0.2 \mathrm{~mm}$ in diameter). If we assume there are 100 primary follicles at any time in a woman of age 20, developing over a course of 4 months, then there is an average of 25 follicles per month leaving the primary pool. The difference between the average decrease in the primordial stage and the average decrease in the primary stage is modeled as atresia in the primordial to primary transition. The difference of 1833 primordial follicles leaving the primordial pool per month, and 25 follicles per month maturing in the primary stage, means approximately $1.4 \%$ of the primordial follicles leaving the primordial pool survive through the primary stage and $98.6 \%$ are lost to atresia. We model this loss as a survival factor in the primordial to primary transition. From this, we have $r_{\text {surv }}=0.014$.

The parameters $r_{2}$ and $a_{1}$ (Eqs. (S2) and (A5)) are correlated with a correlation coefficient of 0.937. The correlation between $r_{2}$ and $a_{1}$ comes from the fact that $a_{1}$ determines the magnitude of $A M H$ in terms of the magnitude of Primar, which is governed by $r_{2}$. Fixing $r_{2}$ during optimization results in unwanted transient behavior in the solution profile for Primar when any of the parameters in the growth term for Primar are changed. For example, decreasing $r_{1}$ without changing $r_{2}$ creates a steep initial drop in the profile for Primar, while increasing $r_{1}$ without changing $r_{2}$ creates a steep initial climb. In order to avoid this transient behavior, we replace $r_{2}$ in the equation for Primar with

$$
r_{2}=\widehat{r}_{2} \cdot \frac{r_{\text {surv }} \cdot r_{1} \cdot 265000}{1+c_{\text {prm }} \cdot 265000+c_{A M H} \cdot a_{1} \cdot 100},
$$

and fix $\widehat{r}_{2}$ during optimization. The value of 0.01 for $\widehat{r}_{2}$ eliminates the unwanted transient behavior (at this value the right hand side of equation (S2) is zero at age 20).

Correlation of parameters in the remaining ovarian system equations (Eqs. (S3)-(S12)) is due in part to the fact that we do not have data for the ovarian stages themselves, but only for the ovarian hormones modeled by the auxiliary equations (A1)-(A4). Theoretically, the follicular stages could grow to any magnitude during optimization, since the auxiliary coefficients ultimately scale them to fit the data. To avoid this, we determine approximate values for the auxiliary coefficients and fix them during optimization. The auxiliary coefficients represent hormone production per ovarian stage volume, and thus can be approximated with knowledge of the hormone levels during the different stages and approximate volumes of each follicular stage. The volume of the dominant follicle at ovulation, $O v$, is taken to be $4000 \mathrm{~mm}^{3}$ assuming that it is approximately a sphere of diameter $20 \mathrm{~mm}$ [27]. Assuming that the ovary is largest around the time of ovulation, we also take $L u t_{1}$ to be $4000 \mathrm{~mm}^{3}$. Then we take the maximum values for the other six follicular stages to differ by increments of $1000 \mathrm{~mm}^{3}$ as follows: $R e F=L_{u} t_{4}=1000, G r F=L_{u} t_{3}=2000$, and DomF $=L u t_{2}=3000$. Finally, we take the 
maximum for $S m A n F$ to be $10 \mathrm{~mm}^{3}$. Assuming these maximum values for the follicular stages, and noting the hormone levels of the data from Welt et al. [16] during these different phases of the cycle, we are able to determine approximate values for the auxiliary coefficients that will result in the necessary hormone levels. The auxiliary coefficients (see Table B.4, Appendix B) are fixed during optimization so that the follicular stages remain at realistic sizes.

Additional correlations exist among parameters for which we have no empirical data. For these correlations, we fix the least sensitive parameters. The parameters $V_{1, L H}$ and $K m_{L H}$ (see Eq. S13) are correlated with correlation coefficient 0.946. $V_{1, L H}$ is the less sensitive parameter, thus we fix it during optimization. The parameter $V_{F S H}$ is correlated with $K i_{F S H, I h A}$ and $K i_{F S H, I h B}$ (see Eq. S15) with correlation coefficients 0.974 and 0.967. $K i_{F S H, I h A}$ and $K i_{F S H, I h B}$ are correlated with correlation coefficient 0.916 . Of these parameters, $V_{F S H}$ is the most sensitive (according to relative sensitivities), thus $K i_{F S H, I h A}$ and $K i_{F S H, I h B}$ are fixed during optimization. The parameters $K m_{F 2}$ and $r_{5}$ are correlated with correlation coefficient 0.988, and $r_{5}$ is more sensitive. The parameters $\omega$ and $c_{5}$ are correlated with correlation coefficient 0.956 , and $\omega$ is more sensitive.

\subsection{Tests for significance}

Tests for significant difference between the model simulations at age 30 versus age 40 (see section 4.2) are performed by using two-tailed two sample t-tests on the means from independent samples of 500 Monte Carlo simulations for each of the two ages. The Monte Carlo simulations are performed by sampling parameters from log-normal distributions with means and standard deviations corresponding to the obtained parameter values and standard errors, respectively (see Appendix B). Only the subset of parameters that are varied during optimization are sampled during Monte Carlo simulation. The means and standard deviations of the simulations are computed separately for each model output (i.e. each hormone or follicular stage), and for each day of the cycle. For each model output there is a family of 28 hypotheses, each corresponding to a day of the monthly cycle. Thus we use the Bonferroni correction [51] to control the overall error rate, i.e., for an overall significance level of $\alpha$, or an overall confidence of $100 \cdot(1-\alpha) \%$, we reject the individual null hypotheses with significance level $\alpha / 28$. We use the significance levels of $\alpha=0.05$ and 0.01 to be significant and very significant, respectively. Hence, in order to achieve overall confidence of $95 \%$ and $99 \%$, we restrict the individual p-values to be less than $0.05 / 28 \approx 0.00179$ and less than $0.01 / 28 \approx 0.000357$, respectively.

\subsection{Model-specific treatment of multiple time scales}

The primordial pool of follicles declines over the lifetime, a timespan of decades. The decline of AMH from mid-reproductive age to menopause follows a similar trend. Data for the primordial pool and AMH are thus on the order of years. The remaining hormones in our model, E2, P4, InhA, and InhB produced in the ovaries and LH and FSH produced in the

pituitary, display daily variations and cycle monthly. The follicular stages that respond to the 
pituitary hormones ( $\operatorname{Pr} A n F$ and subsequent stages, see equations (S3) through (S12)) will also exhibit monthly cycling behavior. Therefore, our model exhibits the time scales of days and of years.

The multiple time scales in this model have the potential of creating numerical and computational difficulties. In order to approximate the daily data of the monthly cycling hormones (E2, P4, InhA, InhB, LH and FSH), the model equations (S1) - (S16) must be solved with a time step less than 1 day. However, to capture the declining trend of the primordial pool and AMH throughout a woman's lifetime, the model equations must be solved over a time span of several decades. Integrating the system from age 20 to age 50 using Matlab's dde23 takes over 8 minutes on a quad-core PC equipped with a generation 7 Intel chip and 8.00 GB RAM. The system of differential equations has 66 parameters, an optimization scheme that integrates the entire system over this time period would take over 8 hours just to change each parameter once, let alone converge to an optimal parameter set. This presents a problem for parameter identification.

In order to use data of the two different time scales in parameter identification for our system, the parameters $a_{2}$ and $a_{3}$ in equation (A5) are set to zero, allowing for the decoupling of equations (S1), (S2), and (A5) from the rest of the system. This system of two ordinary differential equations is solved from age 20 to age 51 using ode23 and optimized against Hansen data and $\mathrm{AMH}_{\text {data }}$ (see Appendix A for residual used during PID).

Once a parameter set is obtained for this small system (see Appendix B for parameter values), the remaining equations (S3)-(S16) and (A1)-(A4) can be solved at any age by using initial conditions for Primor and Primar obtained from the simulation to equations (S1)-(S2) integrated up to the required age. The initial conditions for the remaining state variables (see equations (S3) - (S16)) can obtained at a specific age by fixing Primor and Primar and integrating the remaining equations for two-month time spans until the stable attractor has been reached. Centering the $L H$ peak at day 14, the value of a stage at day 1 is taken to be the initial condition for that stage. When the change in initial condition from one cycle to the next is less than $1 \%$, we assume we have found the stable attractor. The initial conditions for age 20, 30 and 40 are included in Table B.5.

Obtaining parameter values for the remaining parameters involves solving the delay differential equations (S3)-(S16) using dde23 and auxiliary equations (A1)-(A4), for two-month time spans starting at age 30 , i.e., time $t_{30}=30 \times 365$ days and at age 40 , i.e., time $t_{40}=40 \times 365$ days. These solutions are then fit to data for women of ages 20 to 34 and ages 35 to 46, respectively, from Welt et al. [16]. For more information on the residuals used during optimization see Appendix A.

In situations where there are multiple stable attractors for the same parameter set, there is a real possibility that simulations starting at age 40 might settle on a different stable attractor than simulations starting at age 30. For any parameter set tested for this model, numerical 
experiments indicate that there appears to be just one stable attractor for the total time span. Updating the initial conditions at each step during optimization insures that the solution profile is close to this stable attractor. Also, simulation of our model from age 30 through age 40 using the reported parameter set (see Appendix B) is in agreement with the model simulated starting at age 40 with initial condition reported in Appendix B.

Setting $a_{2}$ and $a_{3}$ equal to zero is a simplification of the model that is made to decrease computational cost during parameter identification. However, there is some biological evidence for emphasizing the primary follicle pool. Maciel et al. [24] reported that at any time during the monthly cycle, there are between two and three times as many primary follicles as there are preantral and small antral follicles together. Though individual small antral follicles express more AMH than individual preantral and primary follicles [25], the relative proportions secreted by each cohort is not known.

Since preantral and small antral follicles are cycling monthly, daily levels of AMH throughout the menstrual cycle may be helpful in identifying parameters $a_{2}$ and $a_{3}$ as compared to $a_{1}$. However there is some debate over whether AMH levels exhibit significant daily variability across the menstrual cycle $[52,53]$. Younger women appear to show more variability than older women [54]. In this study we are interested in average monthly AMH, as this is the marker for follicle reserve. We believe that modeling AMH as proportional to the primary follicle count is sufficient for this end.

\section{Simulations and Results}

\subsection{PID of the primordial to primary transition and $A M H$}

Setting $a_{2}$ and $a_{3}$ equal to zero in equation (A5) allows for equations (S1)-(S2) and auxiliary equation (A5) to be decoupled from the larger system, as they no longer depend on the remaining equations. This smaller system is solved from age 20 through age 51 and optimized against the follicle data, Hansen data $_{\text {, }}$ and $\mathrm{AMH}$ data, $\mathrm{AMH}_{\text {data }}$.

The initial condition for the primordial pool is taken from the Hansen et al. [23] (see the equation on page 703) as the power fit to data evaluated at age 20 giving 265,000 follicles. The initial condition for the number of primary follicles at age 20 is taken to be 100 . This value is derived from Broekmans et al. (2009) [5] which asserted that there are between 20 and 150 early growing follicles (sized $0.05 \mathrm{~mm}$ to $2 \mathrm{~mm}$ in diameter) at any time in a woman of age 25 to 40. According to Nussey and Whitehead (2001) [27], preantral and small antral follicles are between between $0.2 \mathrm{~mm}$ and $2 \mathrm{~mm}$ in diameter, and according to Maciel et al. [24] there are between two and three times as many primary follicles as there are preantral and small antral combined. Thus, using the maximum estimate in Broekmans for age 20, we assume there are approximately 100 primary follicles and 50 preantral and small antral follicles at age 20 .

Correlation of parameters was handled as described in Section 3.6. The remaining parameters represent an uncorrelated set, and the numerical optimization algorithm NLSQ_ERR 
applied to this smaller system converges. The optimized parameters for this smaller system are included in Table B.1 of Appendix B. Model output for equations (S1)-(S2) and (A5) are plotted against data in Figure 3.
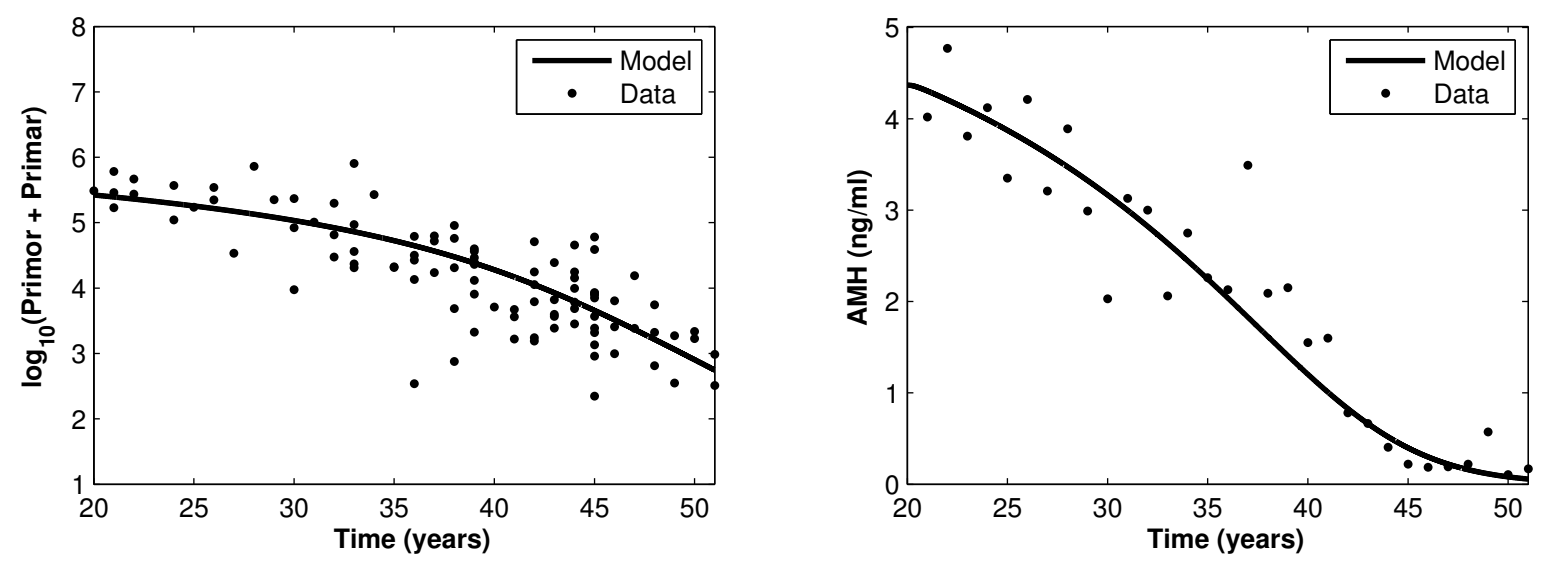

Figure 3: Equations (S1)-(S2) and (A5) are solved using the optimized parameters (Table B.1, Appendix B), starting at age 20 and using the initial conditions Primor $_{0}=265,000$, and Primar $_{0}=100$. The sum of the model solutions Primor and Primar is log transformed and plotted against $\log \left(\operatorname{Hansen}_{\text {data }}\right)$ [23]. The model for $\mathrm{AMH}$ is plotted against composite $\mathrm{AMH}_{\text {data }}[38,17,39,40,41,42]$.

\subsection{PID of monthly cycling follicular stages and hormones, and key changes with age}

Equations (S3)-(S16) can be solved at any age by using initial conditions for Primor and Primar obtained from the solution to equations (S1)-(S2) evaluated at the required age. The simulations for equations $(\mathrm{S} 1)-(\mathrm{S} 2)$ evaluated at age $30\left(t_{30}\right)$ and age $40\left(t_{40}\right)$ give the approximate primordial and primary follicle counts as $\operatorname{Primor}\left(t_{30}\right)=108,000$ and $\operatorname{Primar}\left(t_{30}\right)=72.5$ for age 30, and Primor $\left(t_{40}\right)=19,000$ and $\operatorname{Primar}\left(t_{40}\right)=27.6$ for age 40 . The initial conditions for the remaining stages for a certain age can be obtained by fixing Primor and Primar, which vary little on the time scale of months, and integrating the remaining equations until they have approached the stable attractor. The model simulations are centered with the $L H$ peak at day 14 of the cycle, and day 1 is taken to be the initial condition. This procedure for determining the initial conditions is done whenever the parameter set is varied, and thus must be done at each step in an optimization scheme.

Using the numerical optimization algorithm NLSQ_ERR, we observed that the changes in the logs of the parameters with each iteration converged to less than $10^{-3}$. Since the logs of the parameters were optimized (see Section 3.3), this signifies that the parameters have converged to within $0.1 \%$, and so can be reported to within 3 significant digits. The parameters are reported in Tables B.2, B.3, and B.4. The standard errors (see Section 3.5) associated with this parameter set are also included in Appendix B, and provide an indication of the uncertainty in the presented parameter values. The parameter set reported here provides the smallest 
observed residual. Running 5000 Monte Carlo simulations, sampling parameters from lognormal distributions with means and standard errors as in Appendix B, revealed no parameter set with smaller residual. The simulations obtained from this parameter set are included in Figures 4 to 6 .
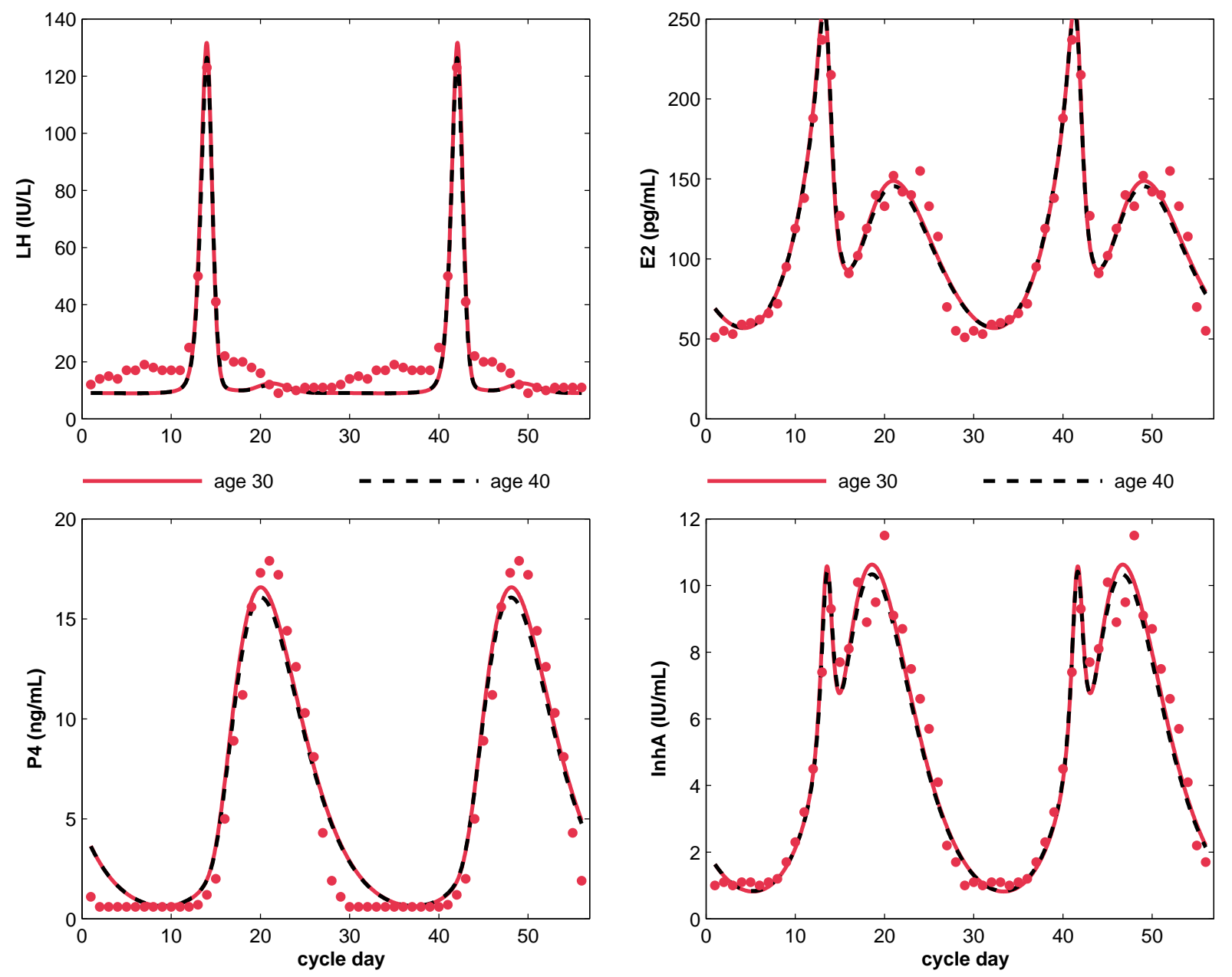

Figure 4: Hormone profiles from the model solved at ages 30 (red solid curves) and 40 (black dashed curves) are plotted against data (red dots) from Welt et al. [16] for women ages 20 to 34 years. Note that the hormone profiles for $L H, E_{2}, P_{4}$ and $I n h A$ are very similar between the two ages. $L H, E_{2}, P_{4}$ and $\operatorname{Inh} A$ are indicative of the ovulatory follicle and corpus luteum, which are similar in ovulatory women of these two age groups [16, 46].

The simulated hormone profiles for $L H, E_{2}, P_{4}$ and $\operatorname{Inh} A$ are plotted in Figure 4 against data for younger women, and the hormone profiles for FSH and $\operatorname{Inh} B$ are plotted in Figure 5 against data from Welt et al. [16] for both younger and older women. Figure 6 contains the solution profiles for the follicular stages $\operatorname{Pr} A n F$ through $L_{u} t_{4}$ (the states associated with equations (S3)-(S12)).

The hormone profiles for $L H, E_{2}, P_{4}$ and $\operatorname{Inh} A$ (Figure 4) are not significantly different for 

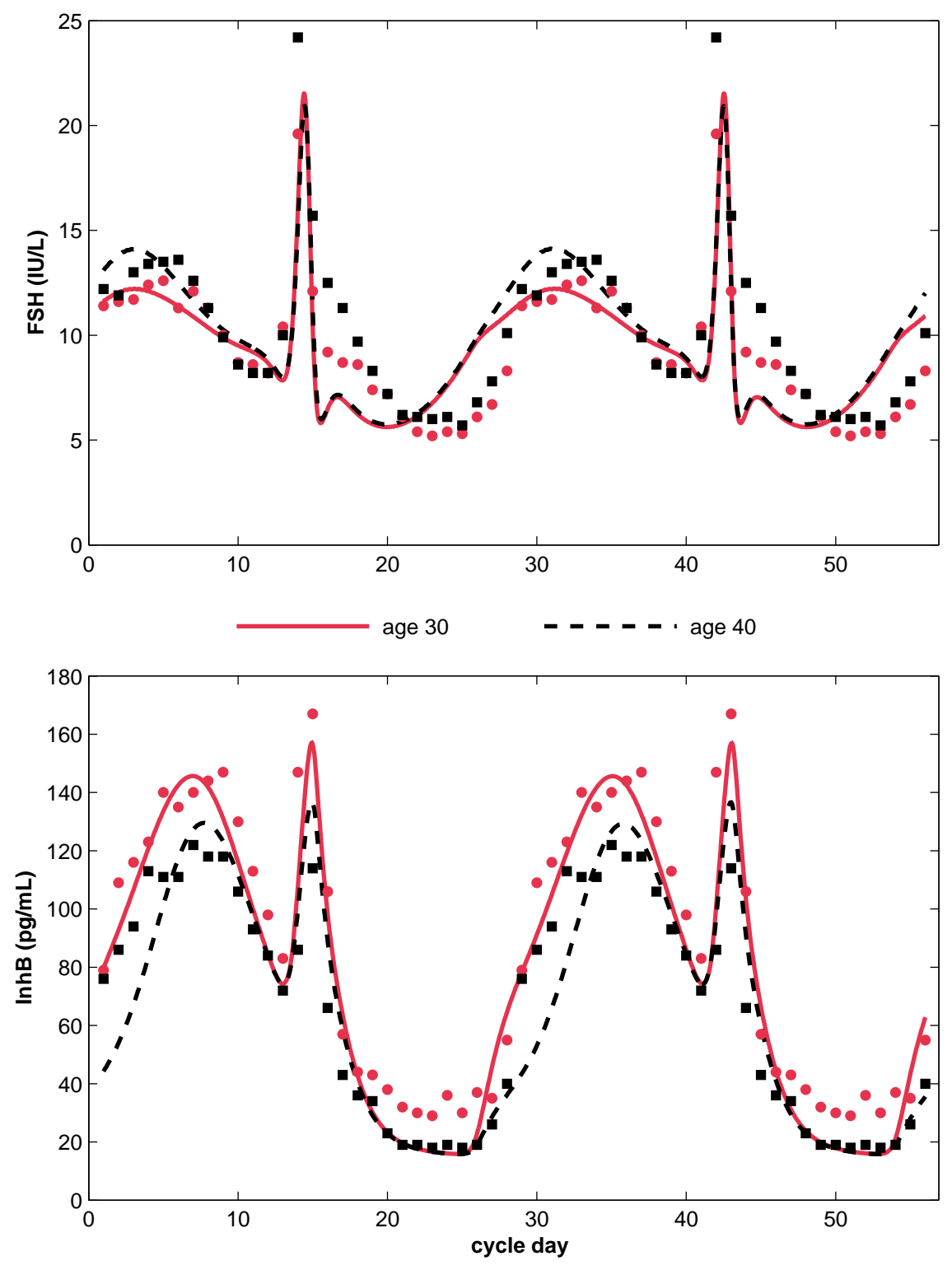

Figure 5: Hormone profiles from the model solved at ages 30 (red solid curves) and 40 (black dashed curves) are plotted against data (red dots) for younger women (ages 20 to 34) and data (black squares) for older women (ages 35 to 46) from Welt et al. [16]. In the older women, early to mid follicular phase (days 1 to 9 ) $\operatorname{Inh} B$ is lower and early to mid follicular phase $F S H$ is higher. $\operatorname{Inh} B$ is produced by early growing follicles which have declined in number between age 30 and 40. The rise in follicular phase $F S H$ is in response to the decreased $\operatorname{Inh} B$. 
PrAnF

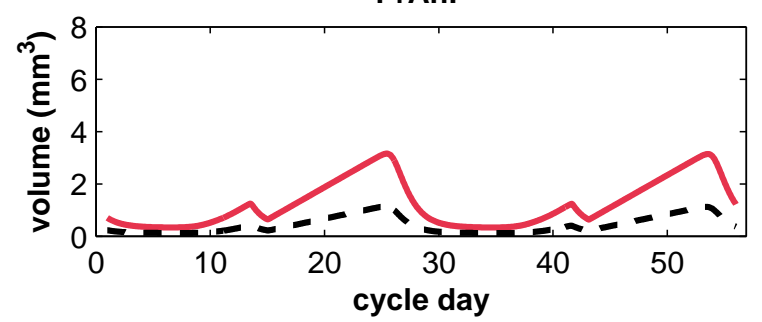

SmAnF

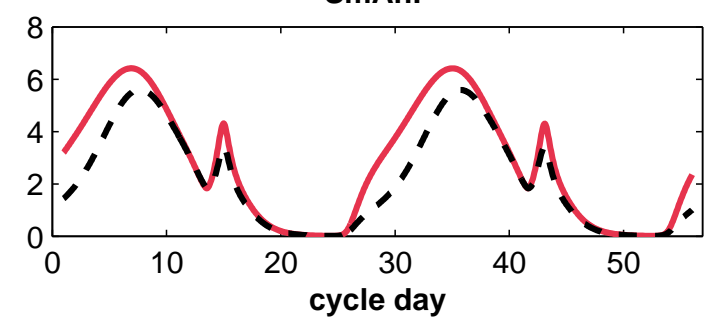

GrF
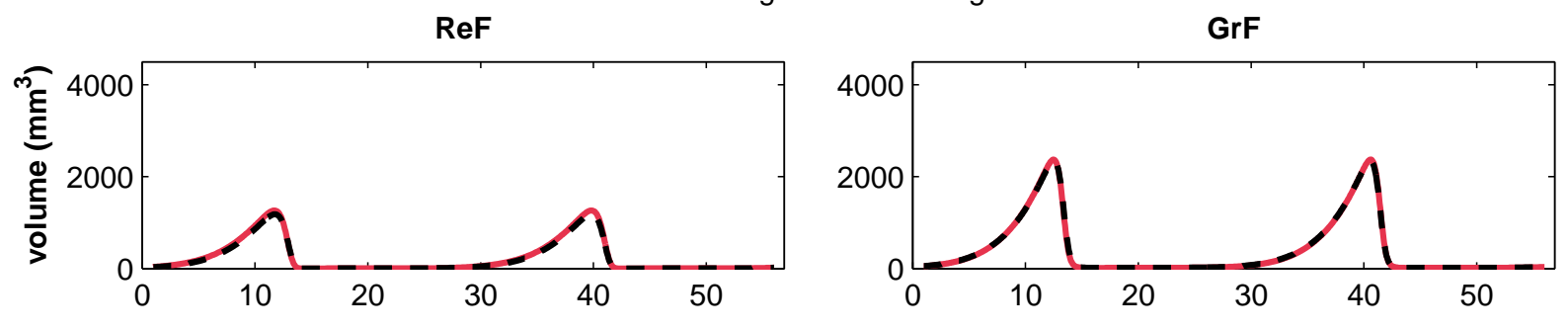

DomF
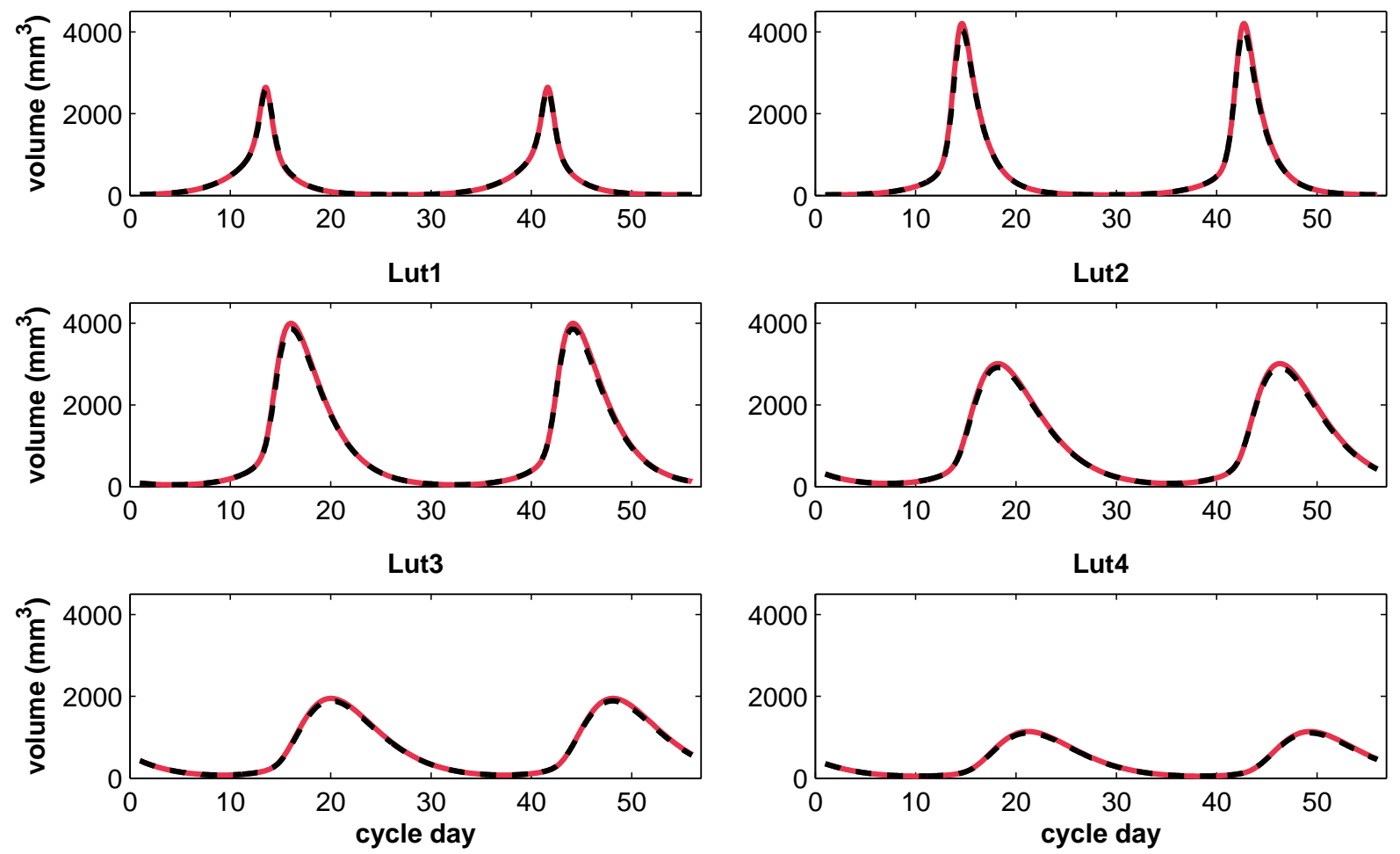

Figure 6: Comparison of follicular stages solved at ages 30 (red solid curves) and 40 (black dashed curves). The solution profiles for $\operatorname{Pr} A n F, S m A n F$, and $R e F$ are significantly different between the two ages (overall confidence 99\%). This is due to the decreased number of primordial follicles that are available to develop into preantral and small antral follicles at age 40. Note that the increased sensitivity to $F S H$ of the growing follicles $(G r F)$ caused by decreased $A M H$ aids in the full development of the dominant follicle and corpus luteum (DomF through Lut4), despite decreased volume of the early antral stages $(\operatorname{Pr} A n F, \operatorname{SmAnF}$, and $\operatorname{ReF})$. 
the two age groups, but $\operatorname{Inh} B$ and $F S H$ (Figure 5) are very significantly different (overall confidence 99\%). The simulations exhibit lower $\operatorname{Inh} B$ and higher FSH during the follicular phase for age 40 as compared to age 30, and these differences are similar to those observed in the Welt et al. [16] data. The Welt data also exhibits differences in luteal InhB. We may be able to model this difference by including additional stages in the definition of InhB. However, since observations reported by others (e.g. van Zonneveld et al. [46]) indicate that luteal InhB is not significantly different between the two age groups, we decided not to complicate the model with features that do not necessarily reflect the physiology.

Inh $B$ is produced by early growing follicles, which have declined in number between ages 30 and 40 (Figure 6), thus the solution profile for $\operatorname{Inh} B$ is lower at age 40 than it is at age 30 (Figure 5). Since $\operatorname{Inh} B$ inhibits $F S H$ synthesis (see eq. (S15)), the decreased InhB causes an increase in follicular phase $F S H$. These differences in $\operatorname{Inh} B$ and $F S H$ simulations correspond to differences in the data (Figure 5). $L H, E_{2}, P_{4}$ and $\operatorname{Inh} A$ are indicative of the ovulatory follicle and corpus luteum which are similar in ovulatory women of these two age groups [46]. Increased sensitivity to FSH of growing follicles caused by decreased $A M H$ (see eq. (S6)) allows for full development of the growing follicles, and thus the dominant follicle, ovulatory follicle and corpus luteum in older women. The volumes of stages $G r F$, DomF through $L_{u} t_{4}$ are not significantly different between age 30 and age 40 (overall confidence 95\%), and this similarity may be observed in Figure 6 . Since these stages contribute to the hormones $E_{2}, P_{4}$ and $\operatorname{Inh} A$ (see eqs. (A1)-(A3)), these hormones are similar between the two age groups and this similarity extends to the $L H$ profiles because $L H$ depends only on $E_{2}$ and $P_{4}$.

\section{Exogenous AMH, AMH agonists and AMH antagonists}

The role that AMH plays in the primordial to primary transition suggests several uses of AMH for fertility treatment, for delaying menopause and for contraception. Since AMH inhibits the transition of follicles from the primordial to the primary stages, AMH or an AMH agonist could be given to premenopausal women to slow this transition and hence may delay the loss of fertility due to low antral follicle count [5]. This treatment would be for women who are waiting to get pregnant until they are older and are worried about the decline of fertility with age due to declining follicle reserve. In the extreme case, if the transition is slowed enough then the number of growing follicles may be decreased enough to prevent ovulation during treatment. Thus AMH or an AMH agonist could be used as a contraceptive. Alternatively, an AMH antagonist could be given to women who are trying to become pregnant but face difficulty due to low antral follicle count. This would be a short term fertility treatment and could possibly be combined with existing fertility treatments such as FSH administration. Our model can be used to simulate outcomes of these treatments. Recall that the numerical optimization algorithms converged to an optimal parameter set for the small system (equations (S1), (S2), and (A5)). Hence the following simulated treatments use an optimal fit to Hansen data and $\mathrm{AMH}_{\text {data }}$ as the 
control.

\subsection{Exogenous AMH Treatment to Delay Menopause}

Predictions for treatment with exogenous AMH from age 25 to 35 with doses that would achieve $5 \mathrm{ng} / \mathrm{mL}$ and $20 \mathrm{ng} / \mathrm{mL}$ increases in serum AMH are included in Figure 7. The treatment is modeled as a constant ( $5 \mathrm{ng} / \mathrm{mL}$ or $20 \mathrm{ng} / \mathrm{mL}$ ) added to the equation (A5) between the ages 25 and 35. The rate of decline of the primordial pool is decreased during the treatment period, and resumes a normal course after the treatment is ended. The number of primary follicles that are developing during the treatment period is decreased, and this decline is dose-dependent. The $5 \mathrm{ng} / \mathrm{mL}$ treatment delays infertility due to low follicle count by 2 years, while the 20 $\mathrm{ng} / \mathrm{mL}$ treatment delays this by 5 years. After the treatment is ended, normal monthly cycling resumes and behaves as it would for a woman 2 or 5 years younger, respectively. If the woman would have stopped ovulating around age 48 without treatment, she would now stop ovulating around age 50, or age 53, respectively. Note that these treatments are not expected to prevent infertility due to factors other than low follicle count.
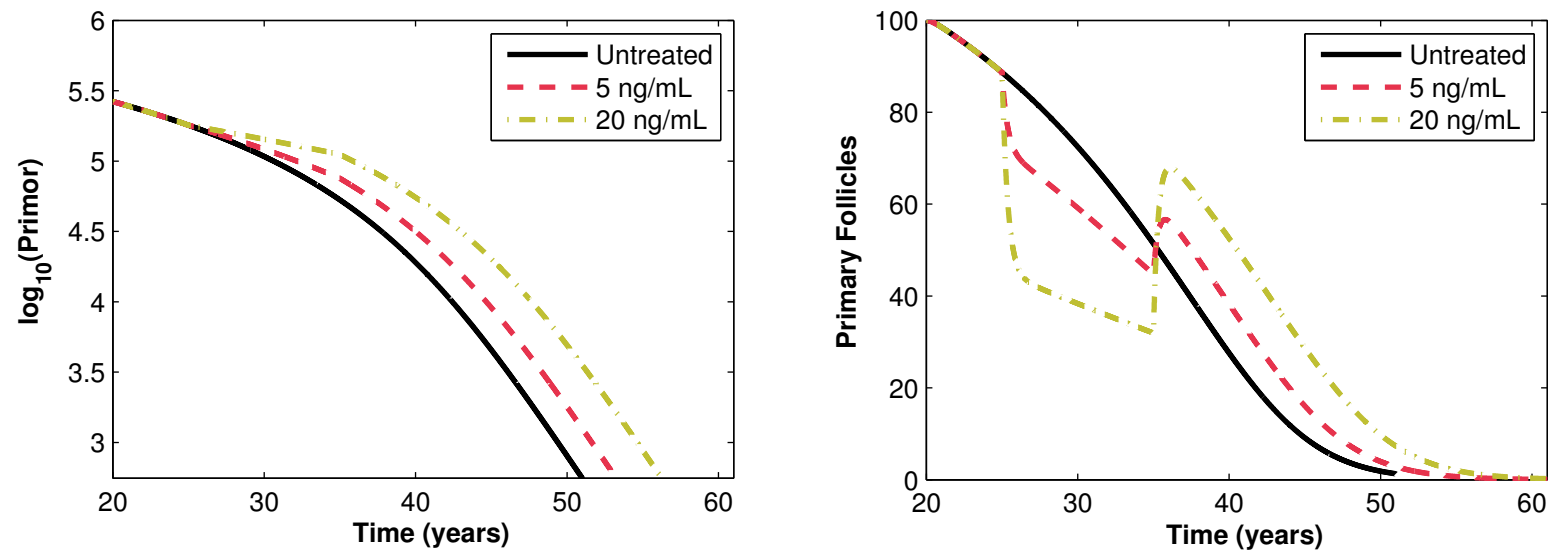

Figure 7: Predicted follicle numbers for individuals given exogenous AMH from age 25 to 35 . Predictions plotted are for treatments that would achieve $5 \mathrm{ng} / \mathrm{mL}$ (red dashed curves) or $20 \mathrm{ng} / \mathrm{mL}$ (yellow dot-dashed curves) increase in serum AMH. The $5 \mathrm{ng} / \mathrm{mL}$ treatment delays infertility due to low follicle count by 2 years and the $20 \mathrm{ng} / \mathrm{mL}$ treatment delays this by 5 years. The number of primary follicles that are developing during the treatment period is decreased, and this decline is dose-dependent.

\subsection{Exogenous AMH Treatment as a Contraceptive}

In theory, if enough $\mathrm{AMH}$ is given during treatment, the number of developing primary follicles would decrease to zero. In order to use AMH as a contraceptive method, the dosage should be large enough to decrease the primary follicle number to a level below what is necessary for ovulation. According to Broekmans et al. 2004 [55], the average age at last child birth (in a population not applying contraceptive measures) is around 41 years. This can be used as a proxy for the age at natural loss of fertility. Broekmans et al. 2009 [5] cites that the average 
age at the onset of cycle irregularity is about 46 years, and the average age at menopause (age at final menstrual period) is 51 years. Our simulations at these ages correspond to primary follicle counts of 23, 7 , and 1, respectively. An AMH treatment that decreases the simulated primary follicle count to below 23 may be sufficient, but a more conservative treatment that decreases it to below 7 or 1 is more likely to prevent ovulation.

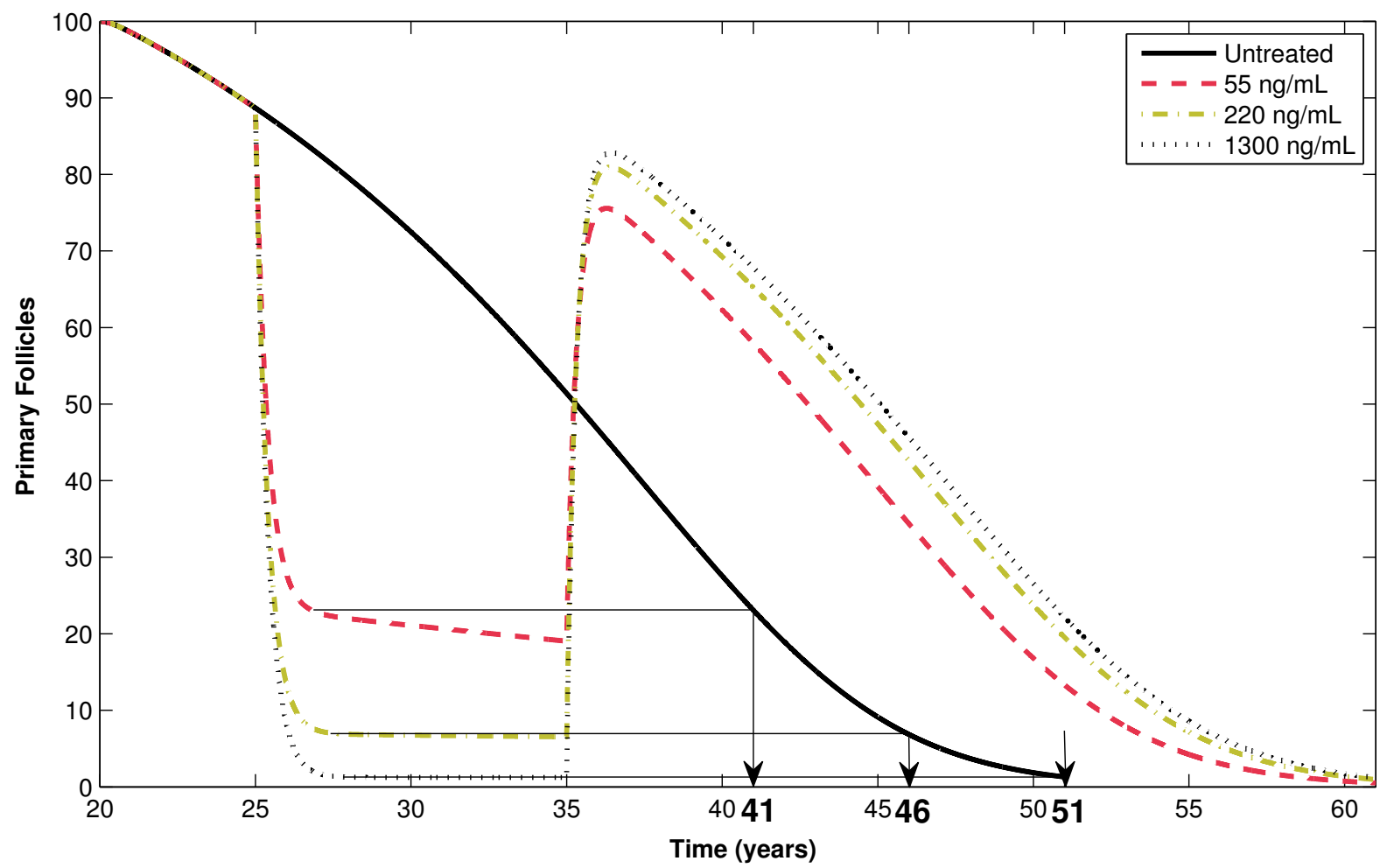

Figure 8: The number of primary follicles is plotted for an AMH contraceptive treatment of $55 \mathrm{ng} / \mathrm{mL}$ (red dashed line), $220 \mathrm{ng} / \mathrm{mL}$ (yellow dot-dashed line), and $1300 \mathrm{ng} / \mathrm{mL}$ (black dotted line) from age 25 to 35 , plotted against the untreated primary follicle profile (black solid line). These are the doses required to decrease the primary follicle count of the average 25 year old to the level of the average 41,46 and 51 year old, respectively. Arrows trace the follicle counts resulting from these treatments across to the untreated curve, and down to the corresponding age on the horizontal axis. These ages correspond to the average ages of natural loss of fertility, onset of cycle irregularity, and menopause, respectively [55, 5].

Our simulation predicts that a dose of $55 \mathrm{ng} / \mathrm{mL} \mathrm{AMH}$ is required to push the primary follicle count of the average 25 year old woman down to that of a woman of age 41 . To decrease the primary follicle count to that of a woman of age 46 and 51, doses of $220 \mathrm{ng} / \mathrm{mL}$ and 1300 $\mathrm{ng} / \mathrm{mL} \mathrm{AMH}$, respectively, are required. Figure 8 plots these treatments given from age 25 to 35. This is a wide range of possible doses required to prevent ovulation. This range could be used as a starting point for determining the therapeutic threshold.

The doses of AMH for possible contraceptive use mentioned here are much higher than 
levels found naturally circulating in women, the first being about 10 times, the second about 50 times, and the third about 300 times the natural level of $\mathrm{AMH}$ in normal mid-reproductive age women. Thus exogenous AMH for the purpose of contraceptive use may be unrealistic. Studies would need to be performed on the effects of AMH on other systems in the body to determine plausibility of exogenous AMH treatments of this magnitude.

\subsection{An AMH Antagonist Fertility Treatment}

Figure 9 includes predictions for treatments with AMH antagonists for one year starting at age 35 or at age 40 where the antagonists block $75 \%$ or $95 \%$ of $\mathrm{AMH}$ action on the primordial to primary transition. The antagonist action is modeled as a factor of 0.25 or 0.05 multiplying the $A M H$ term in the denominator of equations (S1) and (S2). The factor represents the percentage of AMH action not blocked by the antagonist. For the duration of the treatment, the weaker antagonist increases primary follicle numbers by 8 for age 35 (a 16\% increase) and by 3 for age 40 (a $12 \%$ increase). The stronger antagonist increases primary follicle numbers by 10 for age 35 (a 20\% increase) and by 4 for age 40 (a 15\% increase). An AMH antagonist could be used to increase small growing follicle numbers. This could be useful by itself, or as part of other fertility treatments such as exogenous FSH. The AMH antagonist would increase the number of small growing follicles available to respond to FSH. Note that these treatments are not expected to improve fertility in women who experience infertility due to factors other than low follicle count.
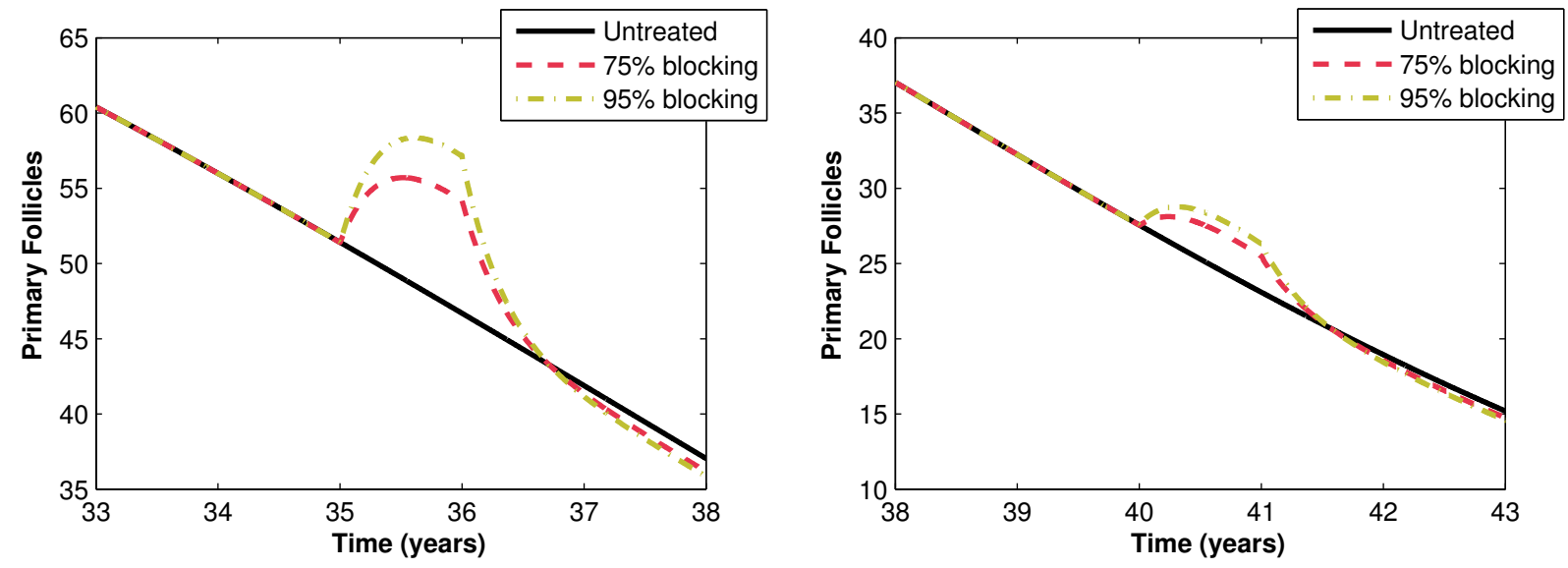

Figure 9: Predicted primary follicle numbers for individuals treated for one year at age 35 (left) and at age 40 (right) with an AMH antagonist that blocks 75\% (red dashed curves) and 95\% (yellow dot-dashed curves) of AMH action on the primordial to primary transition.

\section{Summary and Conclusion}

This study presents a model for hormonal regulation of the menstrual cycle of an adult woman. Our system of 16 nonlinear, delay differential equations with 66 parameters tracks 
normal cycling from a woman's peak reproductive years to menopause. In order to capture age-related changes in hormone levels and in cycle behavior, we model the gradual loss of inactive primordial follicles throughout a woman's life (Figure 3) due to atresia or conversion to the active primary state. The decline in the number of follicles with age results in a noticeable decrease in $A M H$ that begins in a woman's 20's (Figure 3) and a decrease in InhB between ages 30 and 40 (Figure 5). These hormones are produced by preantral and early antral follicles (Figure 6). The drop in $\operatorname{Inh} B$ causes a rise in follicular phase FSH (Figure 5). Levels of $E 2, P 4, \operatorname{Inh} A$ and $L H$ (Figure 4) do not exhibit significant variations between age 30 and 40 primarily because they depend on dominant follicle and corpus luteum development (Figure 6).

In order to obtain the 66 model parameters we develop an ad hoc procedure which results in a model predicting hormonal levels over multiple time scales. This is accomplished by optimizing the parameters of the system $(\mathrm{S} 1)-(\mathrm{S} 2)$ for the primordial and primary follicles. Then the output of the optimized model (S1)-(S2) at any age is used to initiate simulations of the full system (S1)-(S16) at that age (see section 4$)$.

The fact that $\mathrm{AMH}$ inhibits the transition of follicles from the primordial stage to primary stage suggests using model simulations with exogenous AMH for this purpose. Figure 7 shows that treatments with various doses of $\mathrm{AMH}$ may reduce the number of follicles entering the active pool and, hence, delay menopause as measured by the number of primordial follicles remaining in the ovaries. It is not clear if this hypothesis may be investigated clinically. Model simulations show that high amounts of $\mathrm{AMH}$ are needed to reduce active follicle numbers to contraceptive levels (see Figure 8). Finally, Figure 9 shows how an AMH antagonist may temporarily increase the number of small growing follicles which may improve fertility in a woman who is experiencing infertility due to low follicle count.

The age at which simulated cycling in hormone levels ceases seems to be sensitive to parameters and may not represent the actual mechanism of loss of fertility with age. Changes to the model that may account for anovulation and the cessation of cycling would be incorporating thresholds for ovulation and atresia of the dominant follicle if it fails to ovulate. This could be accomplished with a threshold function for LH necessary for ovulation or a threshold for the number of follicles required for dominant follicle selection and ovulation. The latter option would require tracking the numbers of follicles that are developing during the preantral through growing follicle stages. At this point, the model only tracks the numbers of primordial and primary follicles with the remaining follicle stages represented as volumes. Such considerations will be topics of future work.

\section{Acknowledgment}

The authors would like to thank Charles E. Smith for assistance regarding several statistical issues and two anonymous reviewers whose suggestions improved the paper. 


\section{Appendix A - Residuals used during optimization}

The residual vector used in optimization of equations (S1)-(S2) and (A5) is

$$
R=\left[\begin{array}{c}
\frac{R_{P_{o}+P_{a}}}{\sqrt{n_{1}} \max \left(\log \left(\text { Hansen }_{\text {data }}\right)\right)} \\
\frac{R_{A M H}}{\sqrt{n_{2}} \max \left(\mathrm{AMH}_{\text {data }}\right)}
\end{array}\right]
$$

where

$$
R_{P_{o}+P_{a}}=\left[\log (\text { Primor }+ \text { Primar })-\log \left(\text { Hansen }_{\text {data }}\right)\right] \quad \text { and } \quad R_{A M H}=\left[A M H-\mathrm{AMH}_{\mathrm{data}}\right],
$$

and $n_{1}, n_{2}$ are the sample sizes of Hansen $_{\text {data }}$ and $\mathrm{AMH}_{\text {data }}$, respectively. The error for Primor + Primar is computed using log transformed data because the error in the data is consistent with a log-normal distribution. The two residuals $R_{P_{o}+P_{a}}$ and $R_{A M H}$ are divided by the maximum value of the respective data so that these terms are on the same scale. The errors are also each divided by the square root of the number of data points so that the sum of squared residuals of a large and a small data set are weighed equally during optimization.

The residual vector used in optimization of equations (S3)-(S12) and (A1)-(A4) is

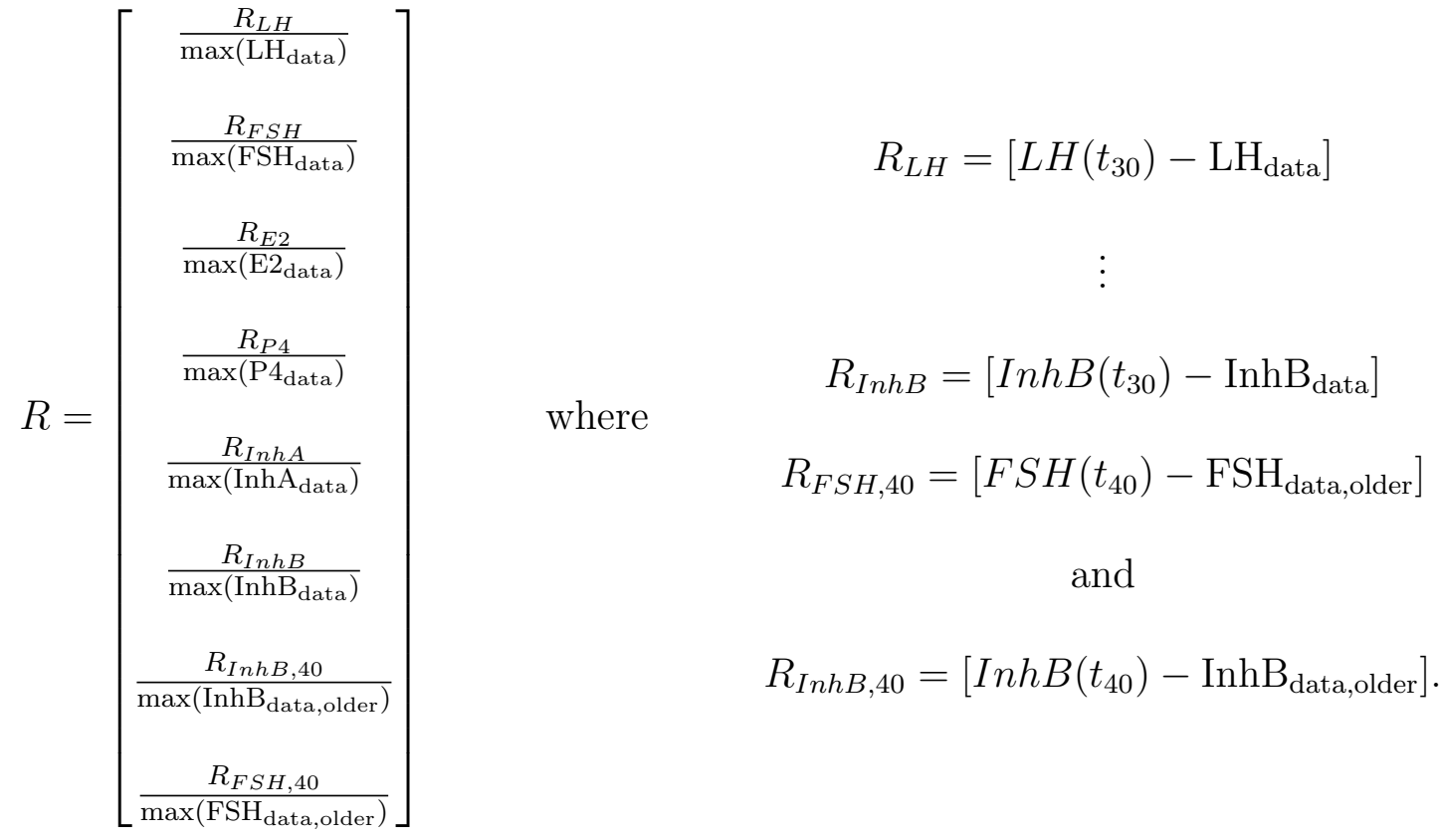

Here $\mathrm{LH}_{\text {data }}, \mathrm{FSH}_{\text {data }}$, etc. are the data for younger women, and $\mathrm{FSH}_{\text {data,older }}$ and $\mathrm{InhB}_{\text {data,older }}$ are the data for older women from Welt et al. (1999) [16]. The residuals include model output of all 6 hormones at age $30\left(L H\left(t_{30}\right), F S H\left(t_{30}\right)\right.$, etc.) compared to data for younger women, and model output for $F S H$ and $\operatorname{Inh} B$ at age $40\left(F S H\left(t_{40}\right)\right.$ and $\left.\operatorname{Inh} B\left(t_{40}\right)\right)$ compared to data for older women. FSH and InhB for older women are included because of the decline of follicular phase $\operatorname{Inh} B$ and subsequent rise in FSH that is seen between age 30 and 40 [16]. 


\section{Appendix B - Parameters and Initial Conditions}

\begin{tabular}{|cll|ll|}
\hline \multicolumn{2}{|c|}{ Eq. (S1)-(S2) } & \multicolumn{2}{c|}{ Eq. (A5) } \\
\hline$c_{A M H}$ & $=0.226 \pm 0.187$ & $\mathrm{~mL} / \mathrm{ng}$ & $a_{1}=0.0437 \pm 0.00463$ & $\mathrm{ng} /(\mathrm{mL}$ follicle) \\
$c_{\text {prm }}$ & $=1.31 \mathrm{E}-05 \pm 2.49 \mathrm{E}-06$ & follicle $^{-1}$ & $a_{2}=0^{*}$ \\
$r_{\text {surv }}$ & $=0.014^{*}$ & & \\
$r_{1}$ & $=0.00102 \pm 0.000178$ & $a_{3}=0^{*}$ & \\
$r_{2}$ & $=0.00694^{*}$ & & & \\
day $^{-1}$ & day $^{-1}$ & & & \\
\hline
\end{tabular}

Table B.1: Optimized parameters for eq. (S1)-(S2) and (A5). This parameter set was obtained by minimizing the sum of square residuals of $\log ($ Primor + Primar $)$ against $\log \left(\operatorname{Hansen}_{\mathrm{data}}\right)$ [23], and $A M H=a_{1}$ Primar against $\mathrm{AMH}_{\text {data }}[38,18,17,39,40,41,42]$ (see Appendix A). The ${ }^{*}$ 's indicate parameter values that were fixed to avoid correlations among parameters during optimization. For $r_{2}$, a scaled version, $\widehat{r}_{2}$, was fixed (see Section 4.1). See Figure 3 for the simulation profiles plotted against data.

\begin{tabular}{|cll|lll|}
\hline \multicolumn{5}{|c|}{ Eq. (S3)-(S12) } \\
\hline vol2 $=0.500^{*}$ & $\mathrm{~mm}^{3}$ & $c_{1}=0.918 \pm 0.0188$ & day $^{-1}$ \\
$K m_{F 1}$ & $=9.82 \pm 0.306$ & $\mathrm{IU} / \mathrm{L}$ & $c_{2}=0.0575 \pm 0.000499$ & $(\mathrm{~L} / \mathrm{IU})^{\delta} /$ day \\
$K m_{F 2}$ & $=10.4^{*}$ & $\mathrm{IU} / \mathrm{L}$ & $c_{3}=0.0241 \pm 0.00172$ & $\mathrm{~L} /($ day IU $)$ \\
$K m_{F 3}$ & $=5.08 \pm 0.355$ & $\mathrm{IU} / \mathrm{L}$ & $c_{4}=0.0307 \pm 0.00299$ & $\mathrm{~L} /($ day IU) \\
$K i_{A M H}$ & $=20.7 \pm 1.37$ & $\mathrm{ng} / \mathrm{mL}$ & $c_{5}=0.198^{*}$ & $(\mathrm{~L} / \mathrm{IU})^{\omega} /$ day \\
$\alpha$ & $=29.1 \pm 2$ & & $c_{6}=0.00519 \pm 5.28 \mathrm{E}-05$ & $\mathrm{~L} /($ day IU) \\
$\beta$ & $=2.06 \pm 0.125$ & & $c_{7}=0.686 \pm 0.0489$ & day $^{-1}$ \\
$\gamma$ & $=2.95 \pm 0.137$ & & $k_{1}=0.416 \pm 0.0258$ & day $^{-1}$ \\
$\delta$ & $=0.999 \pm 0.0245$ & & $k_{2}=0.405 \pm 0.0181$ & day $^{-1}$ \\
$\omega$ & $=0.363 \pm 0.0142$ & & $k_{3}=0.551 \pm 0.0218$ & day $^{-1}$ \\
$r_{3}$ & $=0.759 \pm 0.0657$ & day $^{-1}$ & $k_{4}=0.903 \pm 0.0351$ & day $^{-1}$ \\
$r_{4}$ & $=2.23 \pm 0.0967$ & $\mathrm{~L} /($ day IU) & & \\
$r_{5}$ & $=1.21 \pm 0.0487$ & day $^{-1}$ & & \\
\hline
\end{tabular}

Table B.2: Optimized parameters for equations (S3)-(S12). This list along with the parameters in Tables B.3 and Table B.4 were obtained by minimizing the sum of square residuals of LH, FSH, E2, P4, InhA, and InhB against data from Welt et al. [16] (see Appendix A). The ${ }^{*}$ 's indicate parameter values that were fixed to avoid correlations among parameters during optimization. 


\begin{tabular}{|cll|lll|}
\hline \multicolumn{5}{|c|}{ Eq. $(\mathrm{S} 13)-(\mathrm{S} 16)$} \\
\hline$V_{0, L H}$ & $=343 \pm 16.4$ & $\mathrm{IU} /$ day & $V_{F S H}$ & $=616 \pm 11.9$ & $\mathrm{IU} /$ day \\
$V_{1, L H}$ & $=8110^{*}$ & $\mathrm{IU} /$ day & $K i_{F S H, I n h A}$ & $=2.58^{*}$ & $\mathrm{IU} / \mathrm{mL}$ \\
$K m_{L H}$ & $=247 \pm 4.75$ & $\mathrm{pg} / \mathrm{mL}$ & $K i_{F S H, I n h B}$ & $=120^{*}$ & $\mathrm{pg} / \mathrm{mL}$ \\
$K i_{L H P}$ & $=155 \pm 17.1$ & $\mathrm{ng} / \mathrm{mL}$ & $k_{F S H}$ & $=1.04 \pm 0.103$ & $\mathrm{day}^{-1}$ \\
$k_{L H}$ & $=1.01 \pm 0.0709$ & day & $c_{F S H}$ & $=8.21^{*}$ & $\mathrm{day}^{-1}$ \\
$c_{L H}$ & $=14.0^{*}$ & day & $c_{F S H, P}$ & $=130 \pm 8.61$ & $\mathrm{~mL} / \mathrm{ng}$ \\
$c_{L H P}$ & $=1.10 \pm 0.101$ & $\mathrm{~mL} / \mathrm{ng}$ & $c_{F S H, E}$ & $=0.00525 \pm 0.00058$ & $\mathrm{~mL}^{2} / \mathrm{pg}^{2}$ \\
$c_{L H E}$ & $=0.00398 \pm 7.59 \mathrm{E}-05$ & $\mathrm{~mL} / \mathrm{pg}$ & $d_{I n h A}$ & $=1.38 \pm 0.0852$ & $\mathrm{days}$ \\
$d_{E}$ & $=0.187 \pm 0.0457$ & days & $d_{I n h B}$ & $=0^{*}$ & days \\
$d_{P}$ & $=2.00 \pm 0.249$ & days & $v$ & $=2.5^{*}$ & $\mathrm{~L}$ \\
\hline
\end{tabular}

Table B.3: Parameters for equations (S13)-(S16). The *'s indicate parameter values that were fixed during optimization. Some of these were taken from biological sources, others were fixed at nominal values to avoid correlations among parameters during optimization (see Section 3.6). The parameters $c l_{L H}$ and $c l_{F S H}$ were taken from biological sources [56] and [57]. The parameter $d_{\operatorname{InhB}}$ was taken to be zero as a result of separate analysis of the FSH equations using time-dependent input functions for the ovarian hormones.

\begin{tabular}{|c|c|c|c|c|c|}
\hline \multicolumn{6}{|c|}{ Eq. (A1)-(A4) } \\
\hline$e_{0}$ & $=30$ & $\mathrm{pg} / \mathrm{mL}$ & $h_{0}$ & $=0$ & \\
\hline$e_{1}$ & $=0.04$ & $\mathrm{pg} /\left(\mathrm{mL} \mathrm{mm} \mathrm{m}^{3}\right)$ & $h_{1}$ & $=0.0035$ & $\mathrm{IU} /\left(\mathrm{mL} \mathrm{mm} \mathrm{m}^{3}\right)$ \\
\hline$e_{2}$ & $=0.065$ & $\mathrm{pg} /\left(\mathrm{mL} \mathrm{mm} \mathrm{m}^{3}\right)$ & $h_{2}$ & $=0.0021$ & $\mathrm{IU} /\left(\mathrm{mL} \mathrm{mm} \mathrm{m}^{3}\right)$ \\
\hline$e_{3}$ & $=0.1$ & $\mathrm{pg} /\left(\mathrm{mL} \mathrm{mm^{3 }}\right)$ & $h_{3}$ & $=0.0021$ & $\mathrm{IU} /\left(\mathrm{mL} \mathrm{mm} \mathrm{m}^{3}\right)$ \\
\hline$p_{0}$ & $=0$ & $\mathrm{ng} /\left(\mathrm{mL} \mathrm{mm} \mathrm{m}^{3}\right)$ & $j_{0}$ & $=15$ & $\mathrm{pg} / \mathrm{mL}$ \\
\hline$p_{1}$ & $=0.0085$ & $\mathrm{ng} /\left(\mathrm{mL} \mathrm{mm} \mathrm{m}^{3}\right)$ & $j_{1}$ & $=20.2$ & $\mathrm{pg} /\left(\mathrm{mL} \mathrm{mm} \mathrm{m}^{3}\right)$ \\
\hline$p_{2}$ & $=0$ & $\mathrm{ng} /\left(\mathrm{mL} \mathrm{mm} \mathrm{m}^{3}\right)$ & $j_{2}$ & $=0.0138$ & $\mathrm{pg} /\left(\mathrm{mL} \mathrm{mm} \mathrm{m}^{3}\right)$ \\
\hline
\end{tabular}

Table B.4: Parameters for equations (A1)-(A4). These parameters were obtained by using biologically appropriate magnitudes for the follicular state variables, and estimating the values for the coefficients that would achieve good fits to the data from Welt et al [16] for younger women. These parameter values were fixed during optimization to avoid correlations among parameters. 


\begin{tabular}{|c|c|c|c|}
\hline & Age 20 & Age 30 & Age 40 \\
\hline Primor & 265000 & 108000 & 19000 \\
\hline Primar & 100 & 72.5 & 27.6 \\
\hline $\operatorname{Pr} A n F$ & 1.15 & 0.712 & 0.237 \\
\hline$S m A n F$ & 3.98 & 3.21 & 1.46 \\
\hline$R e F$ & 40.4 & 37.9 & 33.2 \\
\hline$G r F$ & 53.1 & 52.9 & 55.9 \\
\hline DomF & 23.3 & 23.0 & 24.3 \\
\hline$O v$ & 16.0 & 15.6 & 16.3 \\
\hline$L u t_{1}$ & 91.3 & 88.9 & 89.9 \\
\hline Lut $_{2}$ & 320 & 313 & 312 \\
\hline $\mathrm{Lut}_{3}$ & 438 & 429 & 426 \\
\hline $\mathrm{Lut}_{4}$ & 362 & 355 & 352 \\
\hline$R P_{L H}$ & 78.5 & 79.5 & 80.0 \\
\hline$L H$ & 9.05 & 9.05 & 9.05 \\
\hline$R P_{F S H}$ & 12.0 & 12.7 & 14.4 \\
\hline$F S H$ & 11.0 & 11.6 & 13.1 \\
\hline
\end{tabular}

Table B.5: Initial conditions used when solving the model at ages 30 and 40. Initial conditions for Primor and Primar were obtained by solving equations (S1)-(S2) and (A5) from age 20 up to the required age. Initial conditions for the remaining stages were obtained for a specific age by fixing Primor and Primar, and allowing the solution to approach the stable attractor. We consider a less than $1 \%$ change in initial condition from one cycle to the next as a sign that the stable attractor has been reached. Centering the $L H$ peak at day 14 , the value of a stage at day 1 is taken to be the initial condition for that stage. 


\section{References}

[1] S.S.C. Yen, The human menstrual cycle: Neuroendocrine regulation, in: S.S.C. Yen, R.B. Jaffe, and R.L. Barbieri, (Eds.), Reproductive Endocrinology: Physiology, Pathophysiology and Clinical Management. Philadelphia: W.B. Saunders Co., 4th ed., 1999, pp. 191-217.

[2] F.J. Karsch, D.J. Dierschke, R.F. Weick, J. Hotchkiss T. Yamaji, and E. Knobil, Positive and negative feedback control by estrogen of luteinizing hormone secretion in the rhesus monkey, Endocrinol., 92 (1973) 799-804.

[3] M.K. Skinner, Regulation of primordial follicle assembly and development, Hum. Reprod. Update, 11 no. 5 (2005) 461-471.

[4] A.L.L. Durlinger, J.A. Visser, and A.P. Themmen, Regulation of ovarian function: the role of anti-mullerian hormone, Reprod. (2002) 601-609.

[5] F.J. Broekmans, M.R. Soules, and B.C. Fauser, Ovarian aging: mechanisms and clinical consequences, Endocr. Rev., 30 (2009) 465-493.

[6] M.R. Soules, S. Sherman, E. Parrott, R. Rebar, N. Santoro, W. Utian, and N. Woods, Stages of reproductive aging workshop (STRAW), J. Womens Health 8 Gender-Based Med., 10 (2001) 843-848.

[7] R.J. Bogumil, M. Ferin, J. Rootenberg, L. Speroff, and R.L. Vande-Wiele, Mathematical studies of the human menstrual cycle. I. Formulation of a mathematical model, J. Clin. Endocrinol. Metab., 35 (1972) 126-143.

[8] R.J. Bogumil, M. Ferin, and R.L. Vande-Wiele, Mathematical studies of the human menstrual cycle II: Simulation of a model of the human menstrual cycle, J. Clin. Endocrinol. Metab., 35 (1972) 144-156.

[9] L. Plouffe Jr. and S.N. Luxenberg, Biological modeling on a microcomputer using standard spreadsheet and equation solver programs: The hypothalamic-pituitary-ovarian axis as an example, Comput. Biomed. Res., 25 (1992) 117-130.

[10] J.F. Selgrade and P.M. Schlosser, A model for the production of ovarian hormones during the menstrual cycle, Fields Institute Communications, 21 (1999) 429-446.

[11] P.M. Schlosser and J.F. Selgrade, A model of gonadotropin regulation during the menstrual cycle in women: Qualitative features, Environ. Health Perspect., 108 suppl. 5 (2000) 873881. 
[12] L. Harris-Clark, P.M. Schlosser, and J.F. Selgrade, Multiple stable periodic solutions in a model for hormonal control of the menstrual cycle, Bull. Math. Biol., 65 (2003) 157-173.

[13] I. Reinecke and P. Deuflhard, A complex mathematical model of the human menstrual cycle, J. Theor. Biol., 247 (2007) 303-330.

[14] R.D. Pasteur, A multiple-inhibin model for the human menstrual cycle. PhD thesis, North Carolina State University, Raleigh, North Carolina, (2008). http://www.lib.ncsu.edu/resolver/1840.16/5587.

[15] A. Margolskee and J.F. Selgrade, Bifurcation analysis of a model for hormonal regulation of the menstrual cycle with inhibin delay, Math. Biosci., 234 (2011) 95-107.

[16] C.K. Welt, D.J. McNicholl, A.E. Taylor, and J.E. Hall, Female reproductive aging is marked by decreased secretion of dimeric inhibin, J. Clin. Endocrinol. Metab., 84 (1999) 105-111.

[17] M.M. Lee, P.K. Donahoe, T. Hasegawa, B. Silverman, G.B. Crist, S. Best, Y. Hasegawa, R.A. Noto, D. Schoenfeld, and D.T. MacLaughlin, Mullerian inhibiting substance in humans: normal levels from infancy to adulthood, J. Clin. Endocrinol. Metab., 81 (1996) 571-576.

[18] P.L. Hudson, I. Dougas, P.K. Donahoe, R.L. Cat, J. Epstein, R.B. Pepinsky, and D.T. MacLaughlin, An immunoassay to detect human mullerian inhibiting substance in males and females during normal development, J. Clin. Endocrinol. Metab., 70 (1990) 16-22.

[19] G.E. Hale, X. Zhao, C.L. Hughes, H.G. Burger, D.M. Robertson, and I.S. Fraser, Endocrine features of menstrual cycles in middle and late reproductive age and the menopausal transition classified according to the staging of reproductive aging workshop (STRAW) staging system, J. Clin. Endocrinol. Metab., 92 (2007) 2060-3067.

[20] Y.A.R. White, D.C. Woods, Y. Takai, O. Ishihara, H. Seki, and J.L. Tilly, Oocyte formation by mitotically active germ cells purified from ovaries of reproductive-age women, Nature Medicine, 18 no. 3 (2012) 413-421.

[21] S.R. Ojeda, Female reproductive function, in: J.E. Griffin and S.R. Ojeda, (Eds.), Textbook of Endocrine Physiology. Oxford: Oxford University Press, 2nd ed., 1992, pp. 134-188.

[22] W.D. Odell, The reproductive system in women, in: L.J. DeGroot, (Ed.), Endocrinology. New York: Grune \& Stratton, 1979, pp. 1383-1400. 
[23] K.R. Hansen, N.S. Knowlton, A.C. Thyer, J.S. Charleston, M.R. Soules, and N.A. Klein, A new model of reproductive aging: the decline in ovarian non-growing follicle number from birth to menopause, Hum. Reprod., 23 no. 3 (2008) 699-708.

[24] G.A.R. Maciel, E.C. Baracat, J.A. Benda, S.M. Markham, K.Hensinger, R.J. Chang, and G.F. Erickson, Stockpiling of transitional and classic primary follicles in ovaries of women with polycystic ovary syndrome, J. of Clinical Endocrinology \& Metabolism, 89 (2004) $5321-5327$.

[25] J.A. Visser, F.H. de Jong, J.S.E. Laven, and A.P.N. Themmen, Anti-mullerian hormone: a new marker for ovarian function, Soc. for Reprod. and Fertil., 131 (2006) 1-9.

[26] P. Reddy, W. Zheng, and K. Liu, Mechanisms maintaining the dormancy and survival of mammalian primordial follicles, Trends Endocrinol. Metabol., 21 (2009) 96-103.

[27] S.S. Nussey and S.A. Whitehead, Endocrinology: An Integrated Approach. London: Taylor \& Francis, 2001.

[28] A.J. Zeleznik, The physiology of follicle selection, Reprod. Biol. Endocrinol., 2 (2004) 31.

[29] J. Keener and J. Sneyd, Mathematical Physiology I: Cellular Physiology. 2nd ed., New York: Springer-Verlag, 2009.

[30] J.H. Liu and S.S.C. Yen, Induction of midcycle gonadotropin surge by ovarian steroids in women: A critical evaluation, J. Clin. Endocrinol. Metab., 57 (1983) 797-802.

[31] R.I. McLachlan, N.L. Cohen, K.D. Dahl, W.J. Bremner, and M.R. Soules, Serum inhibin levels during the periovulatory interval in normal women: Relationships with sex steroid and gonadotrophin levels, Clin. Endocrinol., 32 (1990) 39-48.

[32] L.F. Shampine and S. Thompson, Solving DDEs in MATLAB, Appl. Numer. Math., 37 (2001) 441-458.

[33] A. Ralston and P. Rabinowitz, A First Course in Numerical Analysis. 2nd ed., New York: Mcgraw-Hill, 1978.

[34] A. Margolskee, ddeRK4, (2012). http://www4.ncsu.edu/ amargol/research/ddeRK4.html.

[35] C.T. Kelley, Iterative methods for optimization, vol. 18 of Frontiers in Applied Mathematics. Philadelphia: SIAM, 1999.

[36] Zuse Institut Berlin: Newton Lib. http://www.zib.de/en/numerik/software/newtonlib.html. 
[37] P. Deufhard, Newton Methods for Nonlinear Problems-Affine Invariance and Adaptive Algorithms. Berlin: Springer-Verlag, 2004.

[38] C.P. Hagen, L. Aksglaede, K. Sorensen, K.M. Main, M. Boas, L. Cleemann, K. Holm, C.H. Gravholt, A. Andersson, A.T. Pedersen, J.H. Petersen, A. Linneberg, S. Kjaergaard, and A. Juul, Serum levels of anti-mullerian hormone as a marker of ovarian function in 926 healthy females from birth to adulthood and in 172 Turner syndrome patients, J. Clin. Endocrinol. Metab., 95 (2010) 5002-5010.

[39] M.R. Sowers, A.D. Eyvazzadeh, D. McConnell, M. Yosef, M.L. Jannausch, D. Zhang, S. Harlow, and J.F. Randolph Jr., Anti-Mullerian Hormone and Inhibin B in the Definition of Ovarian Aging and the Menopause Transition, J. Clin. Endocrinol. Metab., 93 (2008) 3478-3483.

[40] F.R. Tehrani, M. Solaymani-Dodaran, M. Hedayati, and F. Azizi, Is polycystic ovary syndrome an exception for reproductive aging?, Hum. Reprod., 25 (2010) 1775-1781.

[41] R.D. van Beek, M.M. van den Heuvel-Eibrink, J.S.E. Laven, F.H. de Jong, A.P.N. Themmen, F.G. Hakvoort-Cammel, C. van den Bos, H. van den Berg, R. Pieters, and S.M.P.F. de Muinck Keizer-Schrama, Anti-mullerian hormone is a sensitive serum marker for gonadal function in women treated for Hodgkin's lymphoma during childhood, J. Clin. Endocrinol. Metab., 92 (2007) 3189-3874.

[42] J. van Disseldorp, M.J. Faddy, A.P.N. Themmen, F.H. de Jong, P.H.M. Peeters, Y.T. van der Schouw, and F.J.M. Broekmans, Relationship of Serum antimullerian hormone concentration to age at menopause, J. Clin. Endocrinol. Metab., 93 (2008) 2129-2134.

[43] R. Hampl, M. Snajderova, and T. Mardesic, Antimullerian hormone (AMH) not only a marker for prediction of ovarian reserve, Physiol. Res., 60 (2011) 217-223.

[44] D.M. Robertson, G.E. Hale, I.S. Fraser, C.L. Hughes, and H.G. Burger, A proposed classification system for menstrual cycles in the menopause transition based on changes in serum hormone profiles, Menopause: J. North Amer. Menopause Soc., 15 no. 6 (2008) 1139-1144.

[45] H.G. Burger, G.E. Hale, D.M. Robertson, and L. Dennerstein, A review of hormonal changes during the menopausal transition: focus on findings from the Melbourne Women's Midlife Health Project, Hum. Reprod. Update, 13 no. 6 (2007) 559-565.

[46] P. van Zonneveld, G.J. Scheffer, F.J.M. Broekmans, M.A. Blankenstein, F.H.de Jong, C.W.N. Looman, J.D.F. Habbema, and E.R. te Velde, Do cycle disturbances explain the age-related decline of female fertility? Cycle characteristics of women aged over 40 years compared with a reference population of young women, Hum. Reprod., 18 (2003) 495-501. 
[47] H.T. Banks, M. Davidian, and J.R. Samuels, An inverse problem statistical methodology summary, (2007). http://www.ncsu.edu/crsc/reports/ftp/pdf/crsc-tr07-14.pdf.

[48] M. Olufsen and J.T. Ottesen, A practical approach to parameter estimation applied to model predicting heart rate regulation, J. Math. Biol., to appear (2012) .

[49] D.G. Cacuci, Sensitivity and Uncertainty Analysis Theory. Boca Raton: Chapman \& Hall/CRC, 2003.

[50] A.A. Sonin, The physical basis of dimensional analysis. 2nd ed., Dept. of Mech. Eng. MIT, 2001.

[51] R.L. Ott and M. Longnecker, An Introduction to Statistical Methods and Data Analysis. 6th ed., Brooks/Cole Cengage Learning, 2010.

[52] A. La Marca, G. Stabile, A. Carducci Artenisio, and A. Volpe, Serum anti-Mullerian hormone throughout the human menstrual cycle, Hum. Reprod., 21 no. 12 (2006) 31033107.

[53] D.M. Wunder, N.A. Bersinger, M. Yared, R. Kretschmer, and M.H. Birkhauser, Statistically significant changes of antimullerian hormone and inhibin levels during the physiologic menstrual cycle in reproductive age women, Fertil. Steril., 89 (2008) 927-933.

[54] M. Sowers, D. McConnel, K. Gast, H. Zheng, B. Nan, J.D. McCarthy, and J.F. Randolph, Anti-Mullerian hormone and inhibiin B variability during normal menstrual cycles, Fertil. Steril., 94 no. 4 (2010) 1482-1486.

[55] F.J. Broekmans, M.J. Faddy, G. Scheffer, and E.R. te Velde, Antral follicle counts are related to age at natural fertility loss and age at menopause, J. North Amer. Menopause Soc., 11 no. 6 (2004) 607-614.

[56] P.O. Kohler, G.T. Ross, and W.D. Odell, Metabolic clearance and production rates of human luteinizing hormone in pre- and postmenopausal women, J. Clin. Investig., 47 no. 1 (1968) 38-47.

[57] Y.D. Cobel Jr., P.O. Kohler, C.M. Cargille, and G.T. Ross, Production rates and metabolic clearance rates of human follicle-stimulating hormone in premenopausal and postmenopausal women, J. Clin. Investig., 48 (1969) 359-363. 